EGG-NE-10391

\title{
GAS CORE NUCLEAR THERMAL ROCKET ENGINE RESEARCH AND DEVELOPMENT IN THE FORMER USSR
}

Mikhail M. Gurfink

Edited by

\author{
Mervin W. Koehlinger \\ Ralph G. Bennett \\ Chester G. Motloch
}

September 1992

\author{
Idaho National Engineering Laboratory \\ EG\&G Idaho, Inc. \\ Idaho Falls, Idaho 83415 \\ Prepared for the \\ u.S. Department of Energy \\ through the \\ EG\&G Idaho LDRD Program \\ Under DOE Idaho Field Office




\begin{abstract}
Beginning in 1957 and continuing into the mid 1970s, the USSR conducted an extensive investigation into the use of both solid and gas core nuclear thermal rocket engines for space missions. During this time the scientific and engineering problems associated with the development of a solid core engine were resolved. At the same time research was undertaken on a gas core engine, and some of the basic engineering problems associated with the concept were investigated. At the conclusion of the program, the basic principles of the solid core concept were established. However, a prototype solid core engine was not built because no established mission required such an engine. For the gas core concept, some of the basic physical processes involved were studied both theoretically and experimentally. However, no simple method of conducting proof-of-principle tests in a neutron flux was devised.

This report focuses primarily on the development of the gas core concept in the former USSR. A variety of gas core engine system parameters and designs are presented, along with a summary discussion of the basic physical principles and limitations involved in their design. The parallel development of the solid core concept is briefly described to provide an overall perspective of the magnitude of the nuclear thermal propulsion program and a technical comparison with the gas core concept.
\end{abstract}


To Ievlev Vitaly Mikhailovich 


\section{EDITORS' PREFACE}

We are pleased to present this summary report by Dr. Mikhail Gurfink, former head of Fuel Confinement and Thermal Protection in the Gas Core Nuclear Thermal Propulsion Department of the Division of Space Power and Propulsion, Institute of Thermal Processes, Moscow. Dr. Gurfink is now a permanent resident of the United States. His preparation of this report and collaboration with us was funded through a laboratory-directed research and development (LDRD) project at the Idaho National Engineering Laboratory.

Dr. Gurfink has extensive experience in the characterization and investigation of hydrodynamics and heat and mass transfer, specifically as applied to propellant heating, protection of structural components, and flow stability and turbulence suppression in the gas core nuclear thermal propulsion rocket engine. His work has included the theoretical and experimental investigation of the use of magnetic fields to stabilize flow.

In the process of editing this report we have tried to retain the tenor of the original English manuscript prepared by Dr. Gurfink. To the extent possible we have verified the equations and figures and the accuracy of numbers quoted. However, the available information did not permit exhaustive validation of every quantity.

We hope you find this an interesting and informative account of the gas core nuclear thermal propulsion program in the former USSR. Gas core rocket engines involve the understanding of a great number of complex physical phenomena, and the time is right for increased international collaboration and exchange on this area of advanced systems for space propulsion.

Mervin Koehlinger

Ralph Bennett

Chet Motloch 


\section{AUTHOR'S PREFACE}

After a decade and a half of calm in research and development of nuclear thermal propulsion, it is now appropriate to carry out an attentive inventory and analysis of work which has been done during the earlier decades. Such an analysis is especially valuable with respect to gas core nuclear thermal propulsion because of some principally new scientific and technical problems involved, such as the hydrodynamics and neutronics of gaseous fissioning plasmas, the physics of dense plasmas, and the thermal protection of reactor components. Regardless of the difficulties arising in conjunction with the development of gas core nuclear thermal propulsion, the unique performance characteristics of the engine justify the amount of work that has been done and will have to be done. This report is an attempt to present and analyze the work done on gas core rocket engines in the former USSR.

The author considers it a pleasant duty to thank Mr. Chester Motloch, Dr. Ralph Bennett, and Mr. Mervin Koehlinger for their scientific editorial work, and also for their attention to the English style. I am grateful to my son Alexander - he has made me believe that this work was necessary. And special thanks to my wife Tamara for her kind attention and patient help.

Mikhail Gurfink 


\section{CONTENTS}

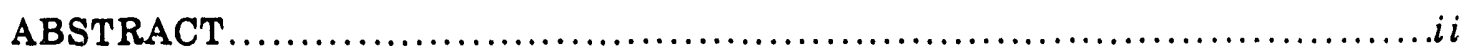

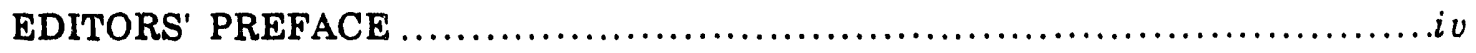

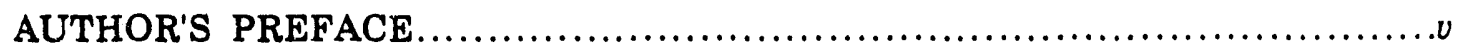

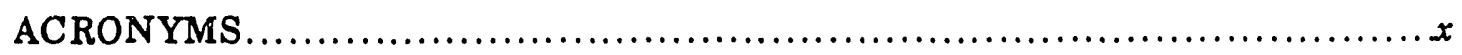

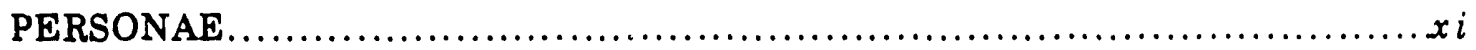

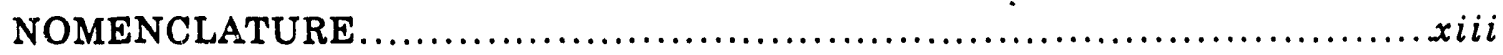

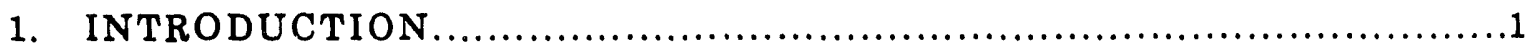

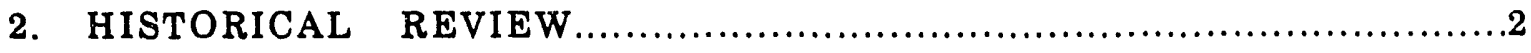

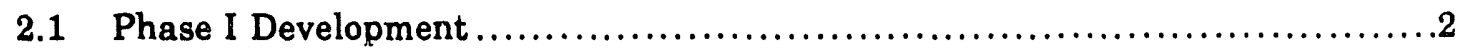

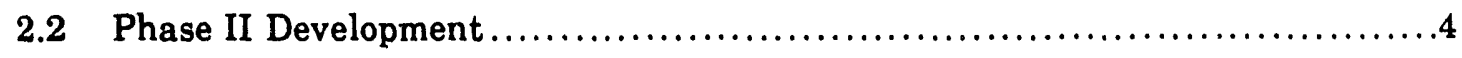

3. ORGANIZATION OF THE DEVELOPMENT WORK.............................

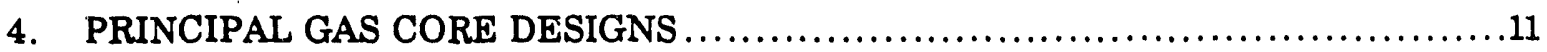

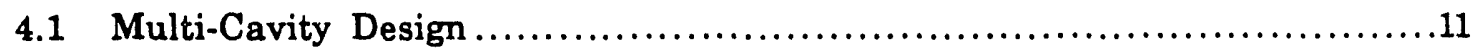

4.2 Single-Cavity Design........................................................

5. REMOVAL OF HEAT GENERATED IN THE MODERATOR ...................16

5.1 Moderator Heat Removal by a Space Radiator ............................16

5.2 Moderator Heat Removal by Regenerative Propellant Flow ..................19

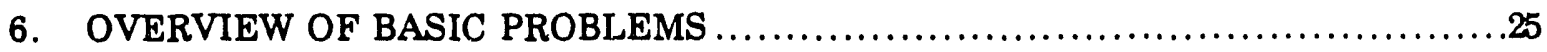

6.1 Entrainment of Fissionable Material ....................................25

6.2 Requirements for Reactor Criticality ...................................28

6.3 Reactor Control and Engine Startup..........................................28

6.4 Properties of Dense Plasmas..................................................

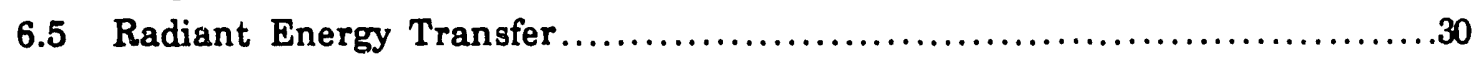

6.6 Thermal Protection of Cavity and Nozzle Walls.............................31

6.7 Acoustic Instability..........................................................

7. CONFINEMENT OF GASEOUS FISSIONABLE MATERIAL ....................33

7.1 Cavity Flow Characteristics......................................................3

7.2 Delaying Transition to Turbulence..................................................

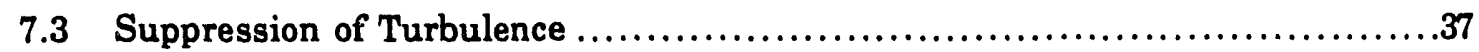

7.4 Increasing Laminar Flow Stability ...............................................

7.5 Effects of Rocket Acceleration on Uranium Loss...........................42

7.6 Using a Magnetic Field to Stabilize Flow..........................................42

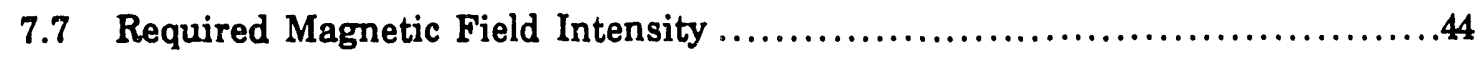




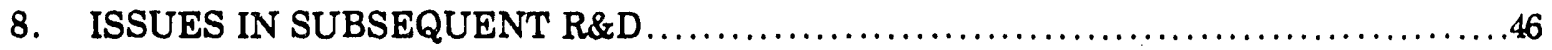

8.1 Current Status........................................................... 46

8.2 Selecting the Design.................................................47

9. COMPARATIVE ASSESSMENT OF SOVIET AND AMERICAN PROGRAMS.......48

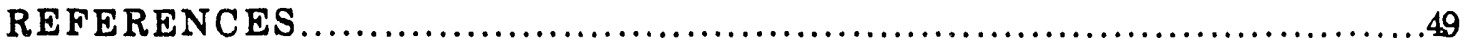




\section{FIGURES}

1. Organization of GCNTP and SCNTP Departments ..........................

2. An Individual Cavity in the Multi-Cavity Gas Core Rocket Engine................12

3. The Single-Cavity Gas Core Rocket Engine .................................

4. Dependence of Thrust and Specific Impulse on Propellant Flow Rate in the Gas Core Rocket Engine with Space Radiator..................................... 18

5. Dependence of Specific Weight and Specific Impulse on Thrust for the Gas Core Rocket Engine with Space Radiator.....................................20

6. Dependence of Specific Impulse on Moderator Outlet Temperature and Propellant in the Gas Core Rocket Engine with Regenerative Cooling..............21

7. Dependence of Specific Weight on Thrust for the Gas Core Rocket Engine with Regenerative Cooling......................................................

8. Reduction of Specific Impulse in a Gas Core Rocket Engine with Entrained Uranium

9. Dependence of Heat Removed by Entrained Uranium on Uranium Retention Time and Thrust.................................................................

10. Dependence of Uranium Entrainment on Reynolds Number $\ldots \ldots \ldots \ldots \ldots \ldots \ldots \ldots .29$

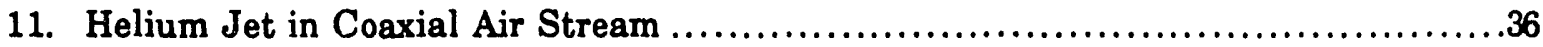

12. Suppressing Effect of a Magnetic Field on Turbulent Flow .......................38

13. Effect of a Longitudinal Magnetic Field on the Mixing Layer Growth in Jet Flow s..................................................................40

14. Dependence of Uranium Retention Time on Magnetic Flux Density and Rocket Acceleration .43 


\section{TABLES}

1. Design Parameters of Three Classes of GCNTP Engines ......................24

2. Range of Reactor Characteristics and Hydrodynamic Parameters in the

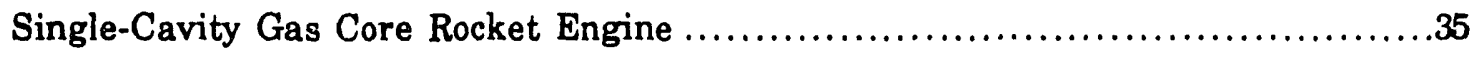

3. Magnetic Field Strengths Required to Stabilize Undesirable Flow Characteristics ...........................................................45 


\section{ACRONYMS}

FEI

GCNTP

GKNT

INEL

LDRD

MHD

MO

MOM

MSM

NII TP

R\&D

RVD

SCNTP

VPK
Physical/Energetics Institute

Gas Core Nuclear Thermal Propulsion

State Committee for Science and Technology

Idaho National Engineering Laboratory

Laboratory Directed Research and Development

Magnetohydrodynamics

Ministry of Defense

Ministry of General Machine Building

Ministry of Medium Machine Building

Institute of Thermal Processes

Research and Development

Transient Research Reactor

Solid Core Nuclear Thermal Propulsion

Military-Industrial Commission 


\section{PERSONAE}

Scientist

Andreyev, V., Dr.

Artamanov, K, Dr.

Barinov, Yu., Eng.

Bogin, V., Dr.

Borisov, N., Dr.

Cheloznov, B., Dr.

Demyanko, Yu., Dr.

Glinnik, R., Dr.

Glushko, V., Acad.

Goldin, A., Dr.

Gorda, V., Dr.

Gorin, A., Dr.

Gurfink, M. M., Dr.

Ievlev, V. M., Dr.

Koba, V., Dr.

Konyukhov, V., Dr.

\section{Responsibility}

Co-head of SCNTP experimental work

Head of GCNTP section, studied acoustic stability of GCNTP engine

Head of development of technological processes for SCNTP fuel elements

Head of SCNTP design and technological work, head of work on powerful magnetic fields

Head of work on GCNTP reactor startup and operation

Co-developer of plasmotron

Co-head of SCNTP experimental work

Supervisor of SCNTP and GCNTP engine design

Member of USSR Academy of Sciences, Head of SCNTP engine design

Design calculations on GCNTP engines

Lead scientist in experimental investigation of SCNTP high-temperature materials

Lead scientist in theoretical estimates applied to problems of SCNTP high-temperature materials and propellants

Head of department of GCNTP fuel confinement and thermal protection, work on magnetohydrodynamics

Corresponding member of USSR Academy of Sciences, head of the department of heat exchange and operational processes, NII TP, lead scientist in GCNTP cavity hydrodynamics and uranium retention

Co-developer of plasmotron

Lead scientist in SCNTP hydraulics and heat transfer 


\section{Scientist}

Koroteyev, A., Dr.

Kostylev, A., Dr.

Kovner, D., Dr.

Krasilnikov, Yu., Dr.

Kuznetsov, V., Dr.

Kuznetsova, N., Dr.

Lappo, N., Dr.

Levin, V., Dr.

Lomovtsev, B., Eng.

Martishin, V., Dr.

Navoznov, O., Dr.

Pavelyev, A., Dr.

Preobrazhensky, S., Dr.

Prishletsov, A., Dr.

Prudnikov, M., Dr.

Solodchenkova, S., Dr.

Treskin, Yu., Eng.

Vanichev, A., Dr.

Zaytsev, V., Eng.

\section{Responsibility}

Head of department of high-temperature modeling: co-developer of plasmotron

Co-developer of plasmotron

Experimental work on the influence of magnetic fields on turbulent flow of a plasma

Experimental work on the influence of magnetic fields on turbulent flow of a plasma

Lead scientist in SCNTP structural design, and cohead of SCNTP experimental work

Co-investigator of performance of GCNTP propellants

Co-investigator of performance of GCNTP propellants

Theoretical work on influence of magnetic fields on turbulent flow of a plasma

Co-developer of plasmotron

Lead scientist in nuclear calculations

Experimental work on delaying transition to turbulence

Experimental work on delaying transition to turbulence

Design calculations on GCNTP engines

Lead s sientist in general calculations of the GCNTP system

Theoretical work on influence of magnetic fields on turbulent flow of a plasma

Co-investigator of performance of GCNTP propellants

Head of SCNTP section

Chair of the Scientific and Technical Council

Co-head of SCNTP experimental work 


\section{NOMENCLATURE}

\section{Symbol Definition}

a Rocket acceleration

c Speed of light

$C_{P} \quad$ Heat capacity of the propellant

$E_{T} \quad$ Strength of thermoeleciric field

F Engine thrust

g Gravitational acceleration

$g_{U} \quad$ Mass fraction of uranium in the propellant

H Magnetic field strength

Ha Hartmann number; ratio of magnetic to

dimensionless viscous forces

$I_{s p} \quad$ Specific impulse

$I_{s p}^{*} \quad$ Characteristic specific impulse (used to non-

dimensionalize the specific impulse)

$I_{s p}$ mix Specific impulse with entrained uranium

$\Delta J \quad$ Energy per unit mass absorbed by the propellant in the reactor

$\Delta \sigma^{*} \quad$ Characteristic energy per unit mass absorbed by the propellant in the reactor (used to non-dimensionalize the corresponding variable, $\Delta N$ )

$m_{f} \quad$ Mass flow rate of propellant

$m_{f} U \quad$ Mass flow rate of entrained uranium

Oe Oersted, a unit of magnetic field strength. In this report, we have generally substituted 1 tesla for every 10,000 Oe, since, for nonmagnetic media, an $\mathrm{Oe}$ is roughly equivalent to a gauss (which is $10^{-4}$ tesla).

Pr Prandtl number

dimensionless

$\mathbf{Q} \quad$ Power emitted by the space radiator 


\begin{tabular}{|c|c|c|}
\hline Symbol & Definition & \\
\hline Re & Reynolds number & dimensionless \\
\hline $\operatorname{Re}_{c r}$ & Critical Reynolds number with a magnetic field & dimensionless \\
\hline $\operatorname{Re}_{\mathrm{cr}}^{\mathrm{O}}$ & Critical Reynolds number without a magnetic field & dimensionless \\
\hline $\operatorname{Re}_{m}$ & Magnetic Reynolds number & dimensionless \\
\hline$S$ & Stuart number; ratio of magnetic to inertial forces & dimension!ess \\
\hline$\Delta T_{M}$ & $\begin{array}{l}\text { Temperature difference between the } \\
\text { moderator inlet and outlet }\end{array}$ & \\
\hline$t$ & Time uranium remains in the core zone & \\
\hline$v$ & Velocity of fluid & \\
\hline$x$ & Distance from the upper cross section of the engine & \\
\hline$\gamma$ & Specific weight & \\
\hline $\boldsymbol{\delta}$ & Width of the mixing layer & \\
\hline$\mu$ & Dynamic viscosity & \\
\hline$\rho$ & Density of fluid & \\
\hline$\sigma$ & Electrical conductivity & \\
\hline$\tau$ & MHD time constant & \\
\hline$\Psi$ & $\begin{array}{l}\text { Ratio of heat released in the moderator to heat added } \\
\text { to the propellant }\end{array}$ & dimensionless \\
\hline$\Psi^{*}$ & Fraction of fission energy released in the moderator & dimensionless \\
\hline$\Psi_{U}$ & $\begin{array}{l}\text { Fraction of fission energy removed by the } \\
\text { entrained and recycled uranium }\end{array}$ & dimensionless \\
\hline
\end{tabular}




\section{GAS CORE NUCLEAR THERMAL ROCKET ENGINE RESEARCH AND DEVELOPMENT IN THE FORMER USSR}

\section{INTRODUCTION}

Space missions undertaken by the Soviet Union have traditionally relied upon chemical propulsion systems for Earth-to-orbit operations and unmanned interplanetary explorations. The specific impulse of the most advanced of such chemical systems is less than $\mathbf{5 0 0}$ sec. However, as early as the late 1950s, it became apparent that future missions (such as orbiting very heavy payloads and conducting interplanetary manned space flights) would require specific impulses greater than $1,000 \mathrm{sec}$. This is a much higher performance level than could reasonably be achieved within the technological and economic framework of rocket engines using chemical propulsion. This recognition led to the early consideration of nuclear propulsion systems.

An extensive investigation into the use of nuclear thermal rocket engines for missions with high propulsion requirements was begun in the Soviet Union in the late 1950s and covered three main areas: investigation of basic feasibility, calculation of engine specifications, and determination of potential applications for nuclear-powered rockets including a comparison with conventional rocket engines. An important element in the research program was the development of conceptual designs of a variety of nuclear thermal propulsion rocket engines. This included characterizing them fairly accurately and determining the range of basic engineering and technological problems.

While work on nuclear thermal rocket engines has been going on in the former Soviet Union for more than three decades, the greatest effort occurred during the period of the 1960 s to the early 1970s. It was during that time that the technologies involved in a nuclear thermal propulsion rocket engine with a solid core were studied and the scientific and engineering problems associated with its practical development were resolved. At the same time research was undertaken to develop the necessary data for a full-scale prototype of a nuclear thermal propulsion rocket engine with a gaseous core, a concept which, theoretically, possesses some attractive characteristics but also presents some difficult engineering problems.

This report is focused primarily on the program surrounding the development of a gas core nuclear thermal propulsion (GCNTP) rocket engine. It describes the overall assessment of the concept, the detailed analyses performed to completely characterize the concept, and the research and development undertaken to solve some basic problems. In addition, the parallel development program on the solid core nuclear thermal propulsion (SCNTP) rocket engine is briefly described to provide an overall perspective and technical comparison with the GCNTP engine. 


\section{HISTORICAL REVIEW}

\subsection{Phase I Development}

In $1957, \mathrm{Dr} . \mathrm{V}$. Ievlev ${ }^{1}$ originated a paper ${ }^{2}$ containing initial estimates of parameters of rocket engines with solid and gaseous cores. In the paper he formulated the basic principles, demonstrated the feasibility of this source of energy, and proposed a research program.

The paper was discussed at a meeting of the Scientific and Technical Council chaired by Dr. A. Vanichev, and it was decided to undertake a more detailed analysis of a variety of designs of nuclear rocket engines, select the most promising concepts, determine the basic parameters of the selected systems, and define the main scientific and technological problems. This work was carried out over the next four years under Dr. Ievlev's supervision.

In the conceptual phase, four fundamentally different systems of increasing complexity were chosen for consideration:

- A system in which the traditional chemical fuel and oxidizer are preheated by a nuclear reactor prior to combustion;

- A system in which a propellant receives thermal energy from a solid core nuclear reactor;

- A system in which a propellant receives thermal energy from a nuclear reactor operating with liquid fissionable material; and

- A system in which a propellant receives thermal energy from a nuclear reactor operating with gaseous fissionable material.

The work done on design definition is described for each of these concepts in turn.

\section{Nuclear-Preheated Chemical Propulsion}

A chemical system with nuclear preheating of the fuel and oxidizer was the natural first step in the development of high specific thrust engines since it was technologically the simplest. Indeed, the temperature required for efficient combustion does not exceed $700 \mathrm{~K}$. At the same time, depending on the physical and chemical properties of the propellant, the specific impulse can be increased 15 to $25 \%$ above that of a conventional rocket engine, a significant increment.

However, calculations showed that the added weight of the nuclear reactor resulted in approximately a ten-fold increase in specific weight (the ratio of weight to thrust) compared to a 
conventional chemical propulsion rocket engine, largely offsetting the advantage of increased specific impulse. The main reason for halting work on this concept was that the conventional rocket engine could be redesigned to reach the same specific impulse without the weight penalty. Furthermore, there seemed to be little possibility of advancing towards more efficient designs and, therefore, little incentive to formulate a convincing long-term plan in which the development of this system would be a priority concern.

\section{Solld Core Nuclear Thermal Propulsion}

Heating propellant in a solid core nuclear reactor was the next logical step towards higher specific impulse. Investigators carried out extensive theoretical and experimental research to select suitable propellants and materials for a high-temperature solid core rocket engine.

During this phase of work the following materials were chosen for the reactor: structural material, graphite; fissionable material, ${ }^{235} \mathrm{U}$; propellant, hydrogen (with small additions of hydrocarbons to reduce corrosion of structural material). Materials considerations limit the operating temperature for this concept to about $2,800 \mathrm{~K}$. The specific impulse is of the order of 900 sec.

Efforts were concentrated on assessing the weight characteristics of the engine and on trajectory calculations pertaining to the placement of heavy payloads in orbit around the earth (requiring large thrust) and to orbital maneuvers and flights to the moon and Mars (requiring low thrust). Calculations on the nuclear reactor to determine the critical payload and weight were carried out in collaboration with the Kurchatov Atomic Energy Institute and $\mathrm{FEI}^{3}$. The basic design was found to be promising and a plan was outlined for the next phase of work.

The leading scientists involved in the development of the SCNTP engine during this and the subsequent phase were Dr. A. Gorin, Dr. V. Gorda, Dr. V. Martishin, Dr. V. Konyukhov, Dr. Dzyubenko, and Eng. V. Kuznetsov.

\section{Llquild Core Nuclear Thermal Propulsion}

Next a system with a liquid core was considered in an attempt to avoid the limitations associated with stability of the solid core reactor structural and fuel materials at high temperatures. In the liquid-core concept heating of the propellant can, in principle, be considered for temperatures greater than $2,800 \mathrm{~K}$, with the operating temperature being determined by the maximum permissible loss of fissionable material as a result of evaporation and entrainment by the propellant.

This system was conceived as a cyclone in which a large number of small liquid particles of fissionable material were suspended in a rotating flow of the propellant. This concept was later 
rejected because the evaporation rate was too high. In addition, the analysis done on this system revealed that this reactor possesses significantly worse reactor physics characteristics than a reactor with solid fissionable material.

\section{Gas Core Nuclear Thermal Propulsion}

The most advanced concept considered was the rocket engine with a gaseous core. The reactor for this system is of the cavity type, i.e., the gaseous fissionable material (plasma core) is located in a spherical or cylindrical cavity surrounded by a moderator. The propellant flows into the cavity between the core and moderator, and heat is transferred from the fissioning material to the propellant largely by thermal radiation and conduction.

A portion of the fission energy generated within the core is deposited in the moderator by attenuation of neutrons and $\gamma$-rays. This energy can be used to regeneratively preheat the propellant before it enters the reactor cavity. For such a design the power output is limited by the allowable moderator temperature. This places a theoretical upper limit on the specific impulse of about 2,900 sec. ${ }^{4}$ Energy removal from the moderator by thermal radiation into space is required to obtain higher specific impulse.

The gaseous state and high temperature of the fissionable material, combined with the density required for criticality of the reactor, leads to a very high pressure in the cavity: $500-800$ atm. More extensive analysis of the design was undertaken, but a significant reduction in the pressure could not be achieved.

After the first reactor physics calculations it became apparent that, due to the significant nonhomogeneity in the cavity, the weight of the reactor would be very large, since a large amount of moderator material is needed to produce criticality. On the other hand, however, the specific weight could be reduced by increasing engine thrust since reactor weight is determined mainly by requirements for criticality, not by hydrodynamics or heat exchange. Thus, relatively high thrust is a characteristic inherent in the GCNTP design.

Participants in the first phase of the gas core work included Dr. V. Ievlev, Dr. V. Martishin, and Dr. A. Prishletsov.

\subsection{Phase II Development}

As a result of the first phase of research, two concepts were selected for further, more detailed work:

- A solid core reactor with specific impulse up to 900 sec; and 
- A gas core reactor with specific impulse up to 2,500 sec.

Beginning in the early 1960s, the main engine parameters for both systems were determined and the basic scientific and technical problems were formulated and investigated.

Over the next few years intensive work was put into formulating and implementing theoretical and experimental work for the purpose of studying the basic processes in both types of nuclear rocket engines. All the work was carried out under the supervision of Dr. V. Ievlev. The solid core section was headed by Engineer. Yu. Treskin; the gas core section was headed by Dr. K. Artamonov.

\section{Solld Core Nuclear Thermal Propulsion}

By the mid 1960s experimental installations had been developed and studies had been carried out on two basic problems of solid core engines: materials interaction between the reactor graphite structure and the hydrogen propellant at working temperatures; and hydraulics and heat exchange in the reactor channels. Experimental data were obtained to develop sufficiently accurate methods of computation, which were then employed to analyze various engine design configurations.

At the same time, test stands were developed for testing graphite blocks in high-temperature flows. Hot gases were produced by an electrical arc heater called a "plasmotron". Individual design features were studied in a series of tests, which yielded promising results. Therefore, it was decided to test a complete fuel element in a neutron flux.

Tests of a graphite element impregnated with ${ }^{235} \mathrm{U}$ were carried out in the early 1970 s jointly with the Kurchatov Atomic Energy Institute on the RVD research reactor, a reactor which has the capability of producing a high neutron flux and transient conditions. This major experimental work concluded an important research phase and demonstrated the basic workability of the unit. It was followed by the development of technology for conducting a full-scale engine ground test in a pilot installation. However, the pilot installation was never built because, at that time, there was no established mission that required just such an engine.

The following people were important contributors to the SCNTP work: Dr. A. Koroteyev, Dr. B. Cheloznov, Dr. A. Kostylev, Eng. V. Koba, Eng. B. Lomovtsev, Dr. V. Bogin, Dr. V. Andreyev, Eng. V. Zaytsev, Dr. Yu. Demyanko, Dr. V. Kuznetsov, Eng. Yu. Barinov, Academician V. Glushko, and Dr. R. Glinnik. 


\section{Gas Core Nuclear Thermal Propulsion}

Research on the gas core reactor began in the mid 1960s with calculation of the range of basic parameters. It was established that, to ensure a critical configuration of the required load of uranium, pressure in the reactor would be of the order of 500 atm and higher. Temperatures of up to $40,000 \mathrm{~K}$ for uranium and $10,000 \mathrm{~K}$ for hydrogen, coupled with high pressure, correspond to dense plasma states, the physics of which had not been researched up to that time. For this reason investigations of thermodynamic and heat transfer properties of dense plasmas were begun. Experimental laborátory installations were built to produce propellants by electric discharge in shock tubes at temperatures in the 5,000 to $20,000 \mathrm{~K}$ range and at pressures in excess of $270 \mathrm{~atm}$.

Data obtained on the physical properties of potential propellants made it possible to refine the calculations of the hydrodynamics and heat exchange in the fuel cavity, mainly for determining the basic structural dimensions, and to undertake very preliminary structural assessments to establish the engine configuration. At that time the basic feasibility of the engine had not yet been proven, though the necessary prerequisites had already been formulated.

During the first phase of development the calculations assumed stationary (non-flow) conditions. It soon became apparent that mixing conditions at the interface between the gaseous uranium and flowing hydrogen had to be laminar or closely approaching it, which was almost impossible to achieve at the actual flow conditions. Therefore, extensive investigations of stability and transition to turbulence were conducted.

Theoretical and experimental studies of the influence of magnetic fields were carried out, and it was shown that magnetohydrodynamic (MHD) effects provided a powerful tool for suppressing turbulence at the interface and reducing mixing in the core. Other experimental work was done on delaying transition to turbulence in jet flows by minimizing any initial disturbance. The methodologies formulated as a result of this work made it possible to carry out a series of calculations and develop designs for practical engines.

The MHD method of flow stabilization in the cavity required determination of the technical feasibility of producing powerful magnetic fields (up to 80 tesla) in large volumes. The study of acoustic stability in the core showed that magnetic fields attainable with modern technology were adequate for completely stabilizing the plasma. Important computational and theoretical work on reactor start-up and operation was also conducted.

By the mid 1970s, when the gas core work began to gradually decline, some of the basic processes in the GCNTP rocket engine had been extensively studied both theoretically and experimentally. Calculational methods had been developed. Several engine designs $\mathrm{h} \cdot \mathrm{d}$ been developed and structurally analyzed, which made it possible to propose future applications of such engines as well as define the principal engineering and technological problems yet to be solved. However, in contrast to work done on the SCNTP engine, no prototype tests in a neutron flux under 
expected operating conditions were undertaken. Furthermore, no sufficiently simple and reliable method of conducting such tests had been devised.

In the decade that followed more detailed research was conducted only in the area of denseplasma physics and hydrodynamics, and calculations and studies were carried out on a simplified method of prototype tests in a neutron flux at zero power. At present virtually all of the necessary scientific and technical data needed to stage a prototype experiment with a gas core reactor are in place.

Contributors to the GCNTP effort were: Dr. V. Ievlev, Dr. N. Kuznetsova, Dr. S. Solodchenkova, Dr. N. Lappo, Dr. M. Prudnikov, Dr. V. Levin, Dr. Ye. Krasilnikov, Dr. D. Kovner, Dr. O. Navoznov, Dr. A. Pavelyev, Dr. R. Glinnik, Dr. A. Goldin, Dr. A. Prishletsov, Dr. S. Preobrazhensky, Dr. V. Bogin, Dr. K. Artamonov, Dr. N. Borisov, and Dr. M. M. Gurfink. 


\section{ORGANIZATION OF THE DEVELOPMENT WORK}

The organizational framework for conducting nuclear thermal propulsion research and development took shape under the constraint that there was not a specific mission for which the engine was being designed. In only two departments was the work on nuclear rocket engines the priority assignment. Within the framework of the Ministry of General Machine Building (MOM) a special section was set up in the Institute of Thermal Processes (NII TP). It was headed by $\mathrm{Dr}$. Ievlev and tasked with a full range of work. Despite its broad scope the work was basically exploratory in nature. Figure 1 shows the established organizational arrangement.

No similar unit was set up within the Ministry of Medium Machine Building (MSM). However, a department was set up in Dr. V. Glushko's design office with the task of designing variants of nuclear rocket engines. The design department was administratively subordinated to Academician Glushko, but functionally it was directly linked with Ievlev's department. Requests for proposals were formulated by NII TP in collaboration with the design department.

To involve other organizations in the work, NII TP employed a "contract" system financed by the Ministry in accordance with annual plans. The hierarchy under the contract system was very simple. The Ministry allocated funding for NII TP, which could use it fairly freely. In this way scientific and research institutes, academic institutions, and technological organizations were drawn into the work.

When drawing up contracts, the scientific definition and technical assignments were formulated by NII TP. Fairly narrow scientific and technological tasks were set, and after completing them the performing organization could cease work; that is, the contracted organizations played a passive role with regard to the problem as a whole.

Another way of developing cooperation was by formulating and issuing so-called "government decrees". The only organization actively formulating such decrees was NII TP. In preparing such decrees the program was coordinated with the Ministry of Defense (MO), the Military-Industrial Commission (VPK), and the State Committee for Science and Technology (GKNT). In this case the work was planned for several years ahead and the decisions were made at the level of several ministries.

Under the government decree system the hierarchy spanned to much higher levels (GKNT, VPK, Council of Ministers) and the involvement of higher echelons was reflected in the fact that when they endorsed a program of work they also provided additional funding for the Ministry of Medium Machine Building and other collaborating ministries. This provided a great motivation for organizations which were passive with respect to the entire problem to get actively involved.

Disregarding studies of individual processes conducted at NII TP, as well as at Academy institutes and universities on a regular basis under NII TP contracts, the sequence of work on the GCNTP engine was as follows. At first NII TP workers made very approximate estimates of 


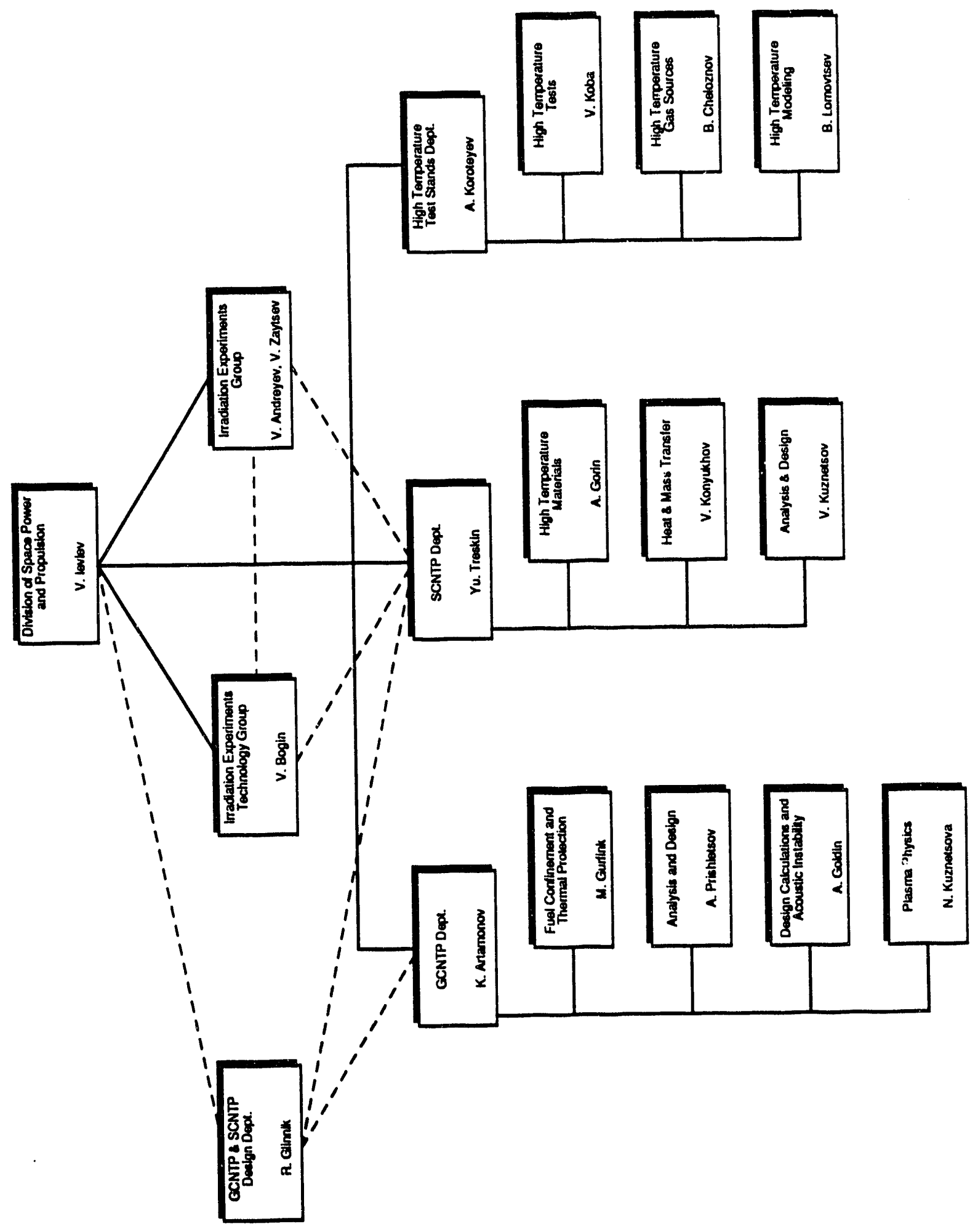

Figure 1. Organization of GCNTP and SCNTP Departments. 
design parameters, such as thrust, specific impulse, weight, dimensions, etc. Then these parameters were used for extensive sensitivity calculations with the purpose of determining the uses of such an engine. After that the Institute formulated a request for research proposals.

Research and develnjment was also carried out by a group of designers from the MOM working in close collaboration with, and under the supervision of NII TP. The task of this group was not the development of a physical device but specification of parameters and determination of engineering ani cechnological problems, i.e., it was part of the R\&D process. All design changes and adjustments were made in direct contact with NII TP, with the blueprints prepared at the conceptual design level. This work made it possible to determine engine performance parameters, which were used for specific second-order trajectory computations.

The Kurchatov Atomic Energy Institute also participated in discussing problems involving nuclear reactors, however, it was not a developer of specific engines. The failure to reach a decision for the development of a gas core engine system brought the work to a halt before the designing and testing of prototypes. 


\section{PRINCIPAL GAS CORE DESIGNS}

Initially a gas core design with several cavities (the most likely number being seven) was proposed. This was a consequence of the early reasoning that because of the large weight of the reactor the GCNTP engine must necessarily have a large thrust, which would be difficult to achieve with a single cavity. The second consideration favoring multiple cavities was the simpler solution this offered for accommodating high pressures and temperatures, owing to the smaller cavity diameter.

In the late 1960s efforts shifted to investigating a single cavity in the wake of failure to clearly establish the need for a high thrust engine. The investigators had also encountered great difficulties with the multi-cavity design, as discussed below. Of the designs described in this chapter, the one drawing the greatest attention was the proposal to use a single-cavity design with a thrust of $200-250 \mathrm{klbf}$ and specific impulse of 2,500 sec for rapid manned flight to Mars.

\subsection{MULTI-CAVITY DESIGN}

Each cavity in the multi-cavity design consists of a prechamber, the cavity proper, and a nozzle. Near the nozzle is an intake for collecting gaseous uranium. Each cavity is hydrodynamically and thermally independent. They are housed together inside the moderator, which they share. The cavity walls are relatively thin and operate in complex thermal and stress conditions. A schematic diagram of an individual cavity is presented in Figure 2.

The design operates as follows: Liquid uranium is injected into the prechamber, which is located in the neutron flux. The purpose of the prechamber is to evaporate the uranium, creating a jet of gaseous fissionable material which is directed into the cavity. The uranium moves along the central axis of the cavity to the recirculation intake. The flow from the recirculation intake together with added hydrogen is directed to the condenser, where most of the condensation occurs. Condensed uranium is pumped back into the prechamber. The initial velocity of the uranium jet is set in the prechamber at the cavity inlet; at the cavity outlet it also depends on the rocket acceleration. The velocity range is $3-5 \mathrm{ft} / \mathrm{sec}$ for a flow section length of 3-5 ft.

Hydrogen propellant flows through the moderator, through the porous walls, and into the cavity, where it is heated to operating temperature and ejected through the nozzle. The mean velocity of the hydrogen increases from $100-150 \mathrm{ft} / \mathrm{sec}$ at injection to $400-600 \mathrm{ft} / \mathrm{sec}$ at the cavity outlet. Hydrogen is also fed to the recirculation intake, providing wall protection and the start of uranium condensation.

Hydrogen must be injected through the walls at a rate sufficient to prevent condensation of uranium on the wall. Since uranium condensation depends on wall temperature, wall 


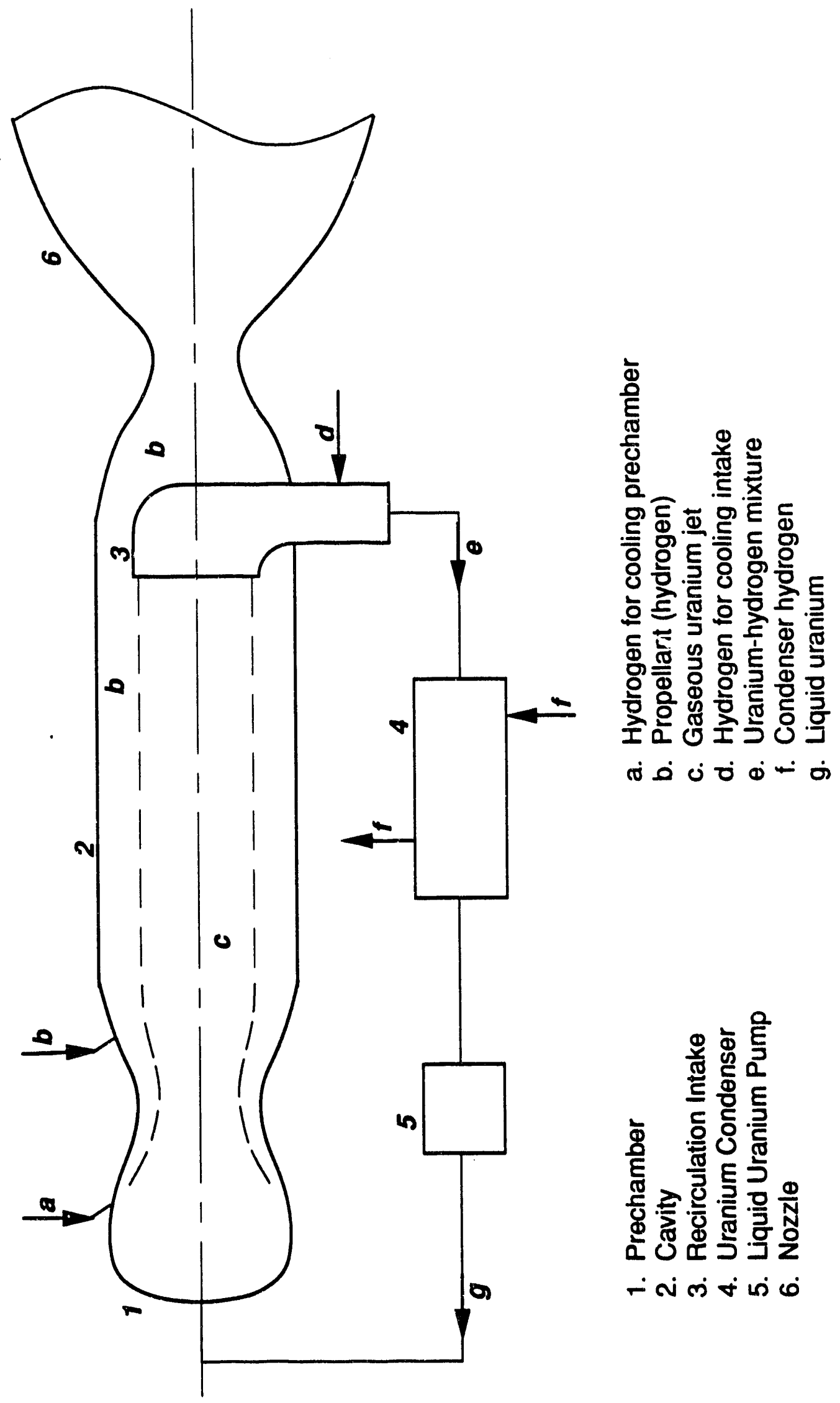

Figure 2. An Individual Càvity in the Multi-Cavity Gas Core Rocket Engine. 
temperature should be as high as possible so that the hydrogen injection rate is adequate. These requirements give rise to the complex technological problem of developing a design with a porous wall made of a refractory metal that can withstand temperatures in the range of $2,000-2,500 \mathrm{~K}$.

Virtually all of the uranium passing through the cavity enters the recirculation intake. Some uranium is lost in the form of vapor and small droplets that are carried out of the nozzle with the propellant flow. Uranium loss is determined, theoretically, by the vapor pressure at the condensate temperature. Thus, this design is very sensitive to the degree of uranium and hydrogen mixing.

The main advantage of the multi-cavity design with uranium recycle is the possibility of easing, if not completely avoiding the main problem of loss of gaseous uranium through the nozzle. However, the basic requirement of this design was very high thrust. This immediately restricts the range of application of such an engine because of launch considerations, with all the attendant safety requirements.

Another advantage of this design is the relatively small diameter of the gaseous uranium jet, which is a result of using several cavities. The re luction in jet diameter leads to an increase in the ratio of surface area to volume, thus providing a large; ihermal radiation surface, a lower temperature gradient in the gaseous uranium, a subrtantial reduction in the mean temperature of the uranium, and a sharp reduction of pressure in the cavity. Estimates yield a pressure range of 200-440 atm, which is quite attainable with modern pressure vessel technology.

The principal difficulty with this design is associated with the uranium recirculation circuit, principally the condenser. To illustrate the magnitude of the provlem, at a thrust of $2 \times 10^{6} \mathrm{lbf}$ and assuming $1 \%$ of the fission energy remains in the uranium as it enters the recirculation intake, the condenser is required tc remove $10^{6} \mathrm{~kW}$ of residual heat plus an additional amount of fission product decay heat, while operating with an outlet temperature greater than $1,000 \mathrm{~K}$. Since no conceptual studies of the system were conducted, the technological or engineering problems connected with the uranium recirculation circuit have not keen investigated. Accordingly, it is also not clear what the practical weight parameters of such an engine system would be.

\subsection{SINGLE-CAVITY DESIGN}

The main feature of the single-cavity design is a single, large cavity containing the fissionable material and propellant surrounded by the moderator. There is no uranium recirculation circuit. Retention of the fissionable material is achieved by hydrodynamic and magnetohydrodynamic methods, while uranium losses are made up ty a special replenishment system. A schematic diagram of a single-cavity design is presented in Figure 3.

The gaseous fissionable material is confined in the middle of the cylindrical cavity. The propellant flows around this central region through a relatively narrow annulus, absorbs energy, 


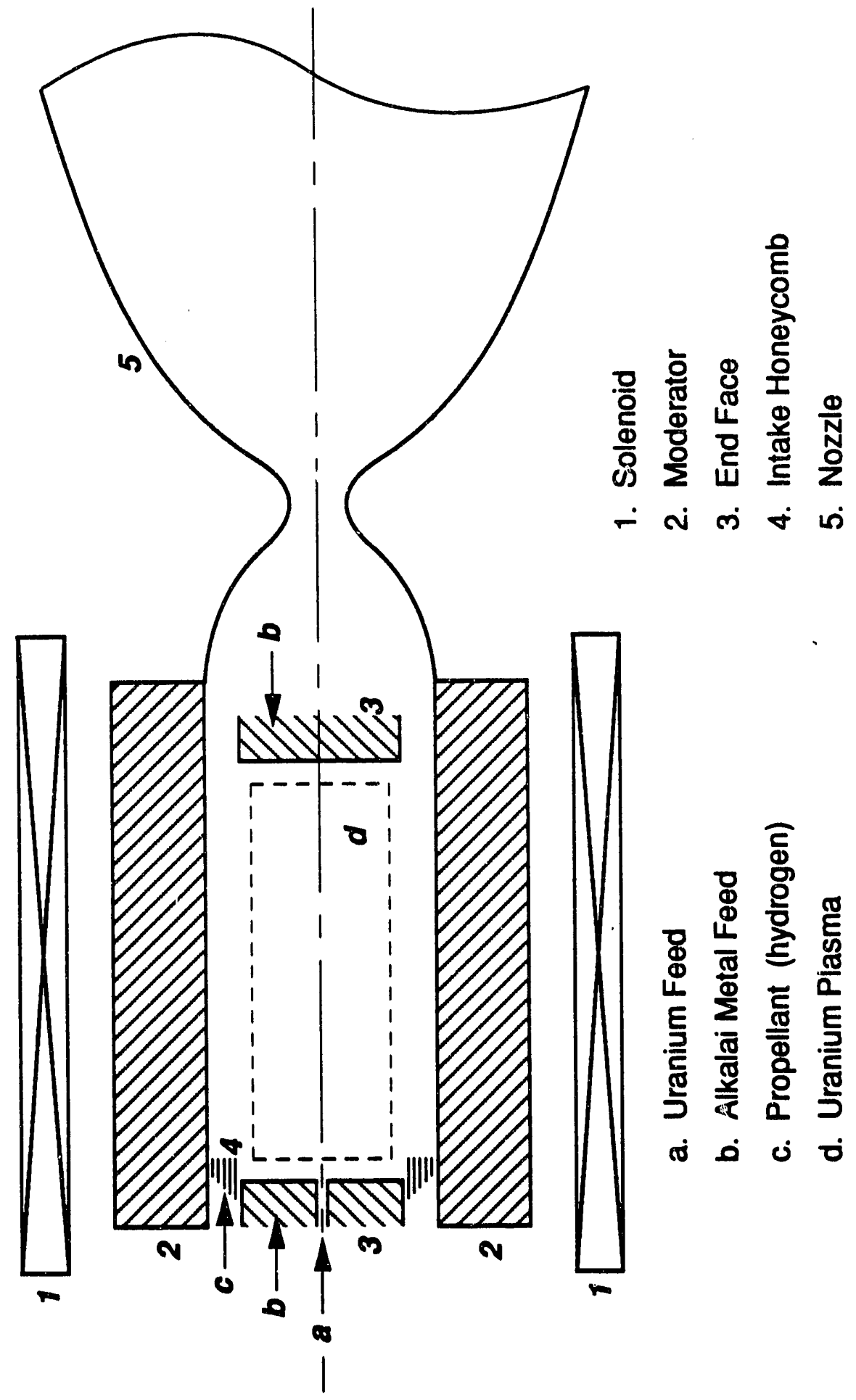

Figure 3. The Single-Cavity Gas Core Rocket Engine. 
and exits through the nozzle. An end face prevents longitudinal displacement of the uranium during rocket acceleration. The uranium is separated from the end surface by a layer of gaseous alkali metal, which reduces radiant heat flow and prevents condensation on the wall. A layer of gaseous alkali metal is also injected between the propellant and the uranium, separating the uranium from the hydrogen and preventing entrainment of the fissionable material by the propellant.

The hydrogen propellant passes through a special honeycomb intake which eliminates turbulent disturbances and also shapes the initial velocity profile, assuring low mixing at the initial section of the cavity where the propellant has not yet heated to a temperature ensuring sufficiently high electrical conductivity. A powerful magnetic field directed along the hydrogen flow is used to confine the gaseous uranium, stabilize the flow of the propellant, and maintain a nearly laminar flow pattern in the cavity. The field is created by a solenoid surrounding the moderator. There are no provisions for uranium recovery, so after passing through the cavity all fluids are ejected through the nozzle.

The main advantage of a single-cavity system is the potential for superior magnetohydrodynamic confinement of the fissionable material. This, however, is achieved by introducing a complex and heavy magnet system, as well as by a significant pressure increase in the cavity. This pressure increase is a consequence of an increase of the diameter of the uranium zone to 2-3 $\mathrm{ft}$ and an associated considerable increase in temperature. In addition, the temperature of the uranium depends on power output, which can vary over a wide range, depending on the purpose of the engine. Extensive calculations yielded a pressure range from 500-1,700 atm. More specific engine parameters are presented in the next two chapters, after a discussion of some basic engine design considerations. 


\section{REMOVAL OF HEAT GENERATED IN THE MODERATOR}

In contrast to the combustion process in a chemical rocket engine, the amount of energy produced by the fission process in a nuclear rocket engine does not directly depend on the nature of the propellant. Most of the fission energy is released in the fuel in the form of the kinetic energy of fission fragments and $\alpha$-particles. However, neutrons and $\gamma$-rays deposit a portion of the fission energy in the moderator, and therefore the moderator must be cooled. Investigations have shown that the method of cooling the moderator has an impact on the specific impulse.

Two methods of cooling the moderator were considered. The first incorporates a specially designed radiator to reject the heat to space. This configuration does not involve the flow of the propellant. The second method is to circulate the propellant through the moderator, thus regeneratively preheating it prior to injecting it into the reactor cavity.

\subsection{Moderator Heat Removal by a Space Radiator}

In this design, the portion of the reactor energy deposited in the moderator is removed by a space radiator. The amount of energy removed can be related to the engine thrust and the propellant flow rate as follows:

Thrust, specific impulse, and propellant flow rate are related by the familiar rocket equation:

$F=I_{s p} m_{f}$

The heat content of the propellant is directly proportional to its temperature, while the specific impulse is directly proportional to the square root of the propellant temperature. Therefore the specific impulse is directly proportional to the square root of the heat content of the propellant, and one can use characteristic values for $I_{s p}$ and $\Delta J$ yielding the relationship:

$\frac{I_{s p}}{I_{s p}^{*}}=\frac{\sqrt{\Delta}}{\sqrt{\Delta J^{*}}}$

Solving for $1_{s p}$ and substituting into Equation 1 yields:

$F=I_{s p}^{*} \frac{\sqrt{\Delta}}{\sqrt{\Delta}} m_{f}$

Now, let $\Psi^{*}$ represent the fraction of the fission energy released in the moderator, and let $\Psi$ be the ratio of heat released in the moderator to heat absorbed in the propellant: 
$\Psi=\frac{\Psi^{*}}{1-\Psi^{*}}$

Then an energy balance on the propellant yields:

$m_{f} \Delta J=\frac{Q}{\Psi}$

Simple algebra yields the final form:

$$
F=I_{s p}^{*} \sqrt{\frac{m_{f}(Q / \Psi)}{\Delta J^{*}}}
$$

When using this equation, characteristic values are assigned to $I^{*}$ sp and $\Delta J^{*}$. The relationship between $F$ and $m_{f}$ can then be determined for various values of $Q / \Psi$. Finally, $I_{s p}$ is calculated using Equation 1. According to this equation the thrust is proportional to the square root of the propellant flow rate. The results of computations using this relationship are presented in Figure 4 for three values of radiator power.

It can be seen from the curves that, owing to comparatively lower power, the design with space radiator may prove to be more efficient (in terms of specific impulse) than the solid core engine (for which $I_{s p}=800 \mathrm{sec}$ ) for thrust in the range of 10-30 klbf, and comparable to a gas core without a radiator (for which $I_{s p}=2,500 \mathrm{sec}$ ) for thrust in the range of $7-12 \mathrm{klbf}$. The design with a space radiator can yield higher specific impulse than the arrangement without radiator at thrust in the range of 2-5 klbf.

Note also that:

$F \cdot I_{s p}=I_{s p}^{*} \sqrt{\frac{m_{f}(Q / \Psi)}{\Delta J^{*}}} \cdot I_{s p}^{*} \sqrt{\frac{(Q / \Psi) / m_{f}}{\Delta J^{*}}} \propto Q / \Psi$

This demonstrates that limitations imposed on the capacity of a space radiator also leads to corresponding limitations on thrust or specific impulse.

The foregoing analysis should be complemented with a consideration of the weight characteristics of a gas core reactor. Numerous calculations, which have been confirmed in comparisons with the results of critical assemblies, have shown that a gas core reactor is significantly heavier than a solid core reactor. This is due to the relative positions of the fissionable material and the moderator, the large quantity of neutron-absorbing structural materials, and the heavy reflector and housing which must be of considerable thickness and 


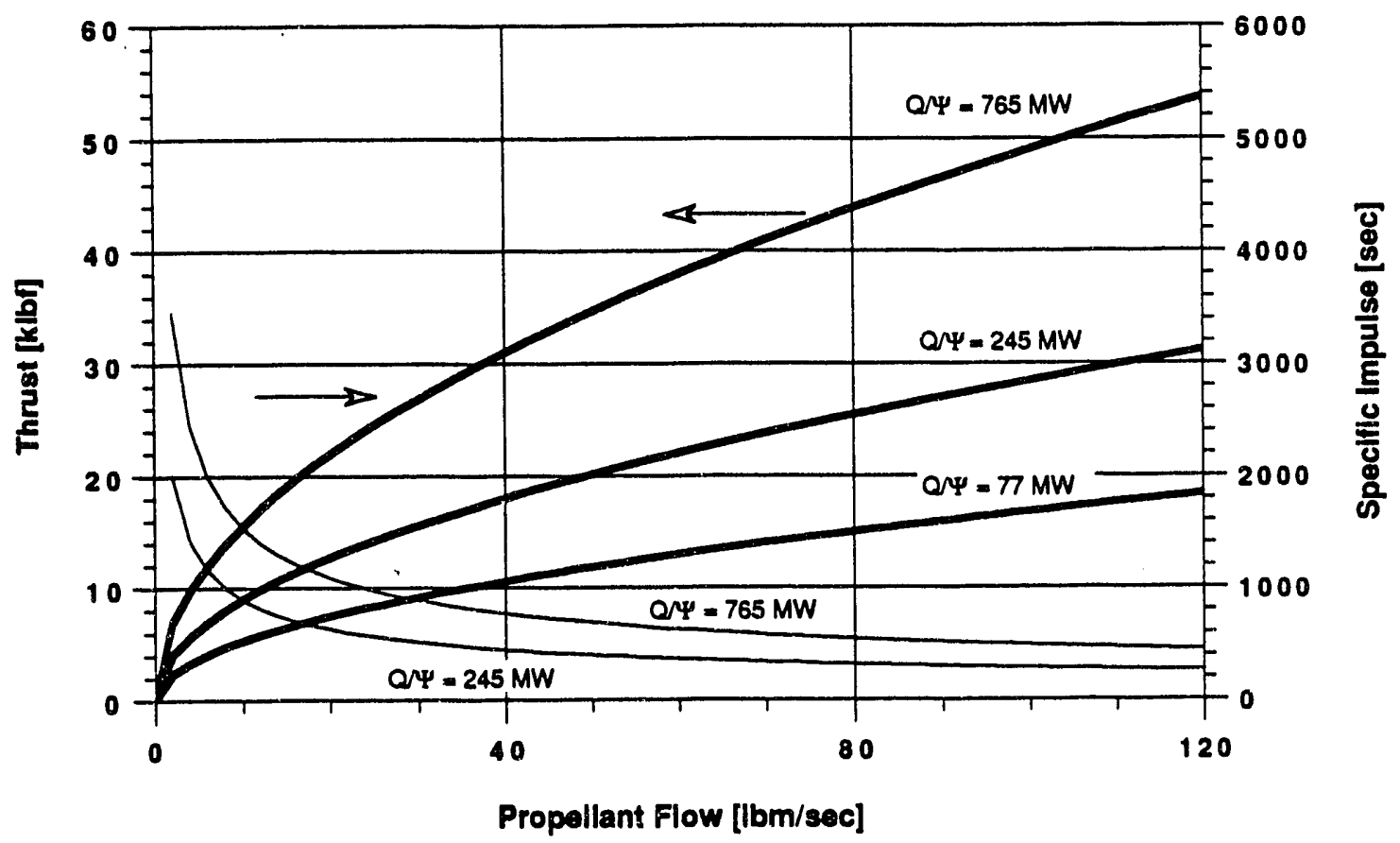

Figure 4. Dependence of Thrust and Specific Impulse on Propellant Flow Rate in the Gas Core Riocket Engine with Space Radiator. 
withstand high operating pressures. The use of a magnetic system for confinement also increases weight.

It is important to note that the reactor weight is determined mainly by considerations of neutron physics, not engine design. Numerous calculations and design estimates at the conceptual level have demonstrated that the mass of a graphite reactor with a beryllium oxide reflector is in the 40-50 tonne range. It is especially important to stress that, in the case of a gas reactor, its weight is only weakly dependent on the engine thrust. F'or that reason engine specific weight (weight-to-thrust ratio) is virtually in inverse proportion to the thrust.

Figure 5 presents the dependence of the specific weight, $\gamma$, and the specific impulse of a gas core engine on thrust for two typical values of $Q / \Psi$. It can be seen that the engine weight considerably exceeds the thrust and, moreover, the increase in specific impulse is directly linked with lower thrust, and hence with increased specific weight. Such an engine cannot be used for launching, only for interplanetary propulsion; moreover, we repeat, its weight is great regardless of thrust.

The foregoing study indicates the basic trend of increasing specific impulse with the reduction in thrust inherent in this arrangement. Indeed, at a thrust below $5 \mathrm{klbf}$, Figure 5 shows an incredible specific impulse for this type of engine: up to 5,000 sec. In this connection it is necessary to note that an increase in the specific impulse of the engine inevitably leads to a sharp pressure increase inside the reactor. Indeed, the temperature to which the hydrogen is heated increases in proportion to the square of the specific impulse, which results in an approximately linear increase in the temperature of the gaseous fissionable material. From the condition of the need to place the critical load in the cavity, it therefore follows that the pressure in the reactor must increase accordingly. Tentative calculations carried out at NII TP indicate that at a specific impulse of 5,000 sec, pressure in the reactor reaches $900-1,800$ atm, which is at the very limit of technical possibilities in the foreseeable future, at least as applied to rocket engines.

\subsection{Moderator Heat Removal by Regenerative Propellant Flow}

Consider $\Psi^{*}$, the fraction of fission energy released in the moderator, to be a constant value. Then the heat content of the propellant at the reactor outlet, $\Delta J$, is given by:

$\Delta=\frac{C_{P} \Delta T_{M}}{\Psi^{*}}$

Thus, the heat content depends on the propellant's heat capacity and the allowable temperature of the moderator material. In other words, in this case the energy generated in the reactor is not independent of the type of propellant, which makes the choice of hydrogen virtually mandatory. This is shown in Figure 6, which presents the dependence of specific impulse on the temperature of the propellant at the moderator outlet for both hydrogen and methane. 


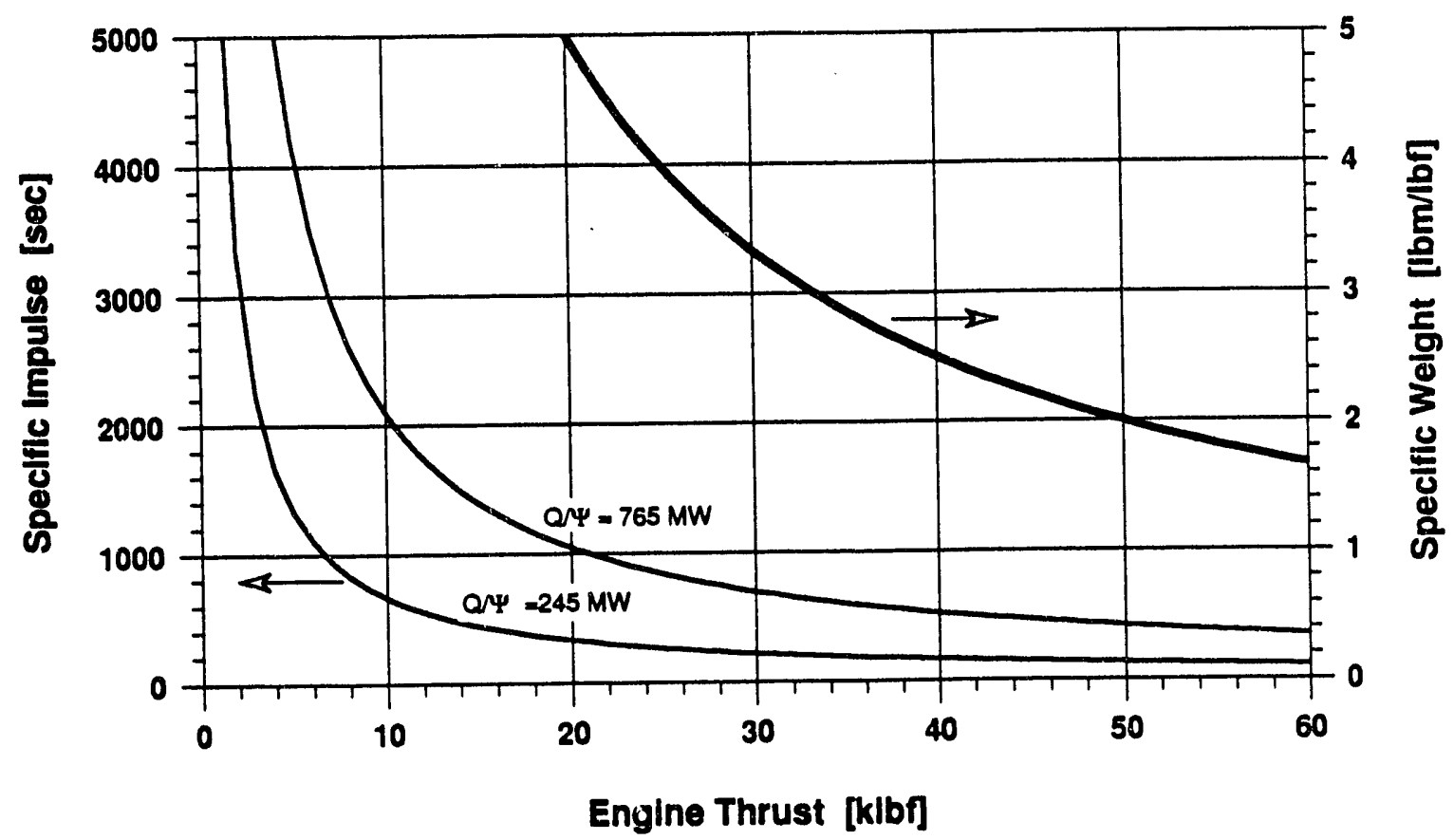

Figure 5. Dependence of Specific Weight and Specific Impulse on Thrust for the Gas Core Rocket Engine with Space Radiator. 


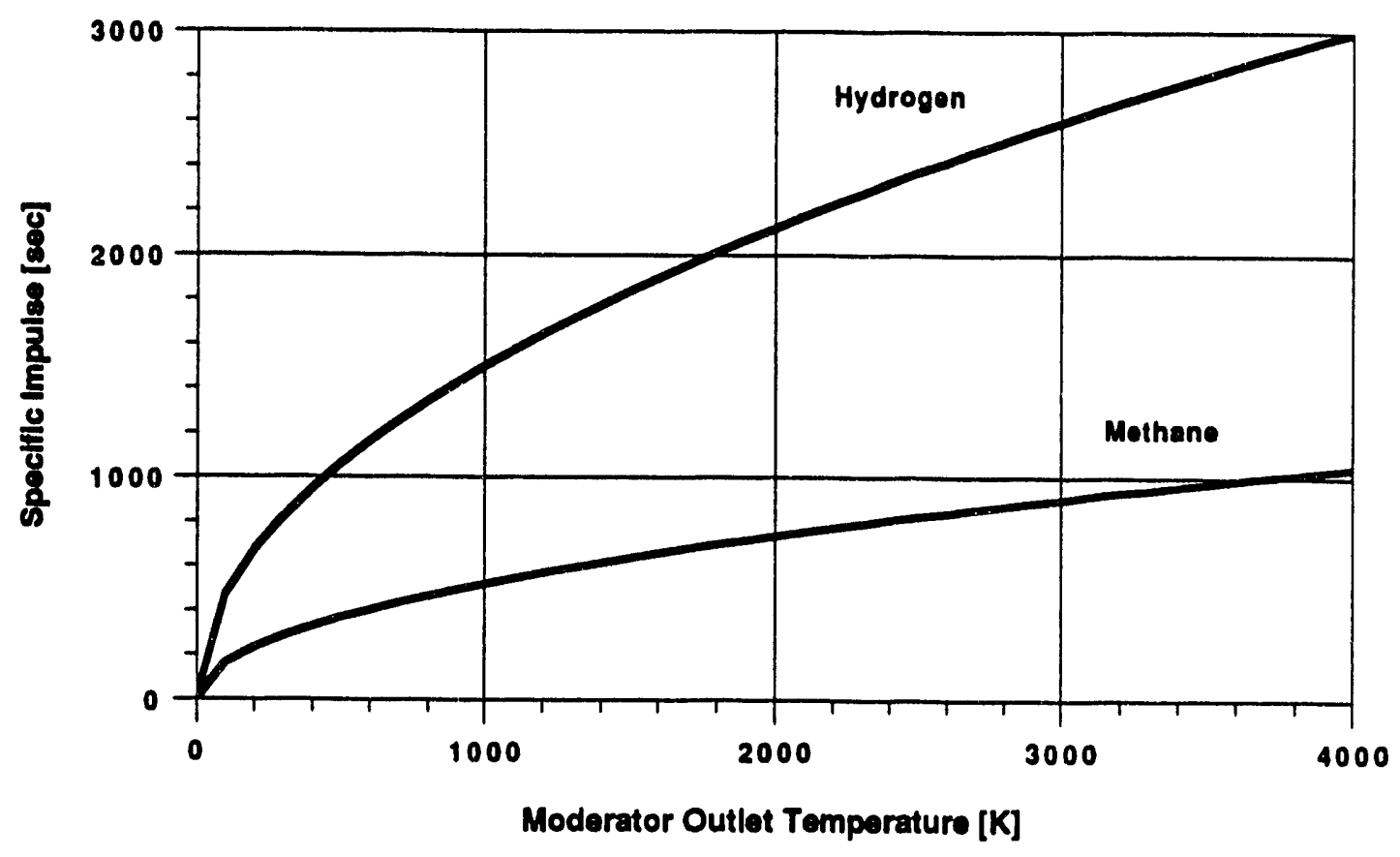

Figure 6. Dependence of Specific Impulse on Moderator Outlet Temperature and Propellant in the Gas Core Rocket Engine with Regenerative Cooling. 
The foregoing leads us to the concept of "limiting specific impulse", which is determined by the maximum achievable heating of the hydrogen in the moderator. Numerous computations for different arrangements yield a value of $I_{s p}=2,500 \mathrm{sec}$, which corresponds to a maximum heating temperature in the moderator of $2,800 \mathrm{~K}$. Thus, the high-temperature moderator in a GCNTP engine is fairly similar to the core of an SCNTP engine.

In this regenerative arrangement, the thrust can vary within a wide range, with no limitations set by the value of the specific impulse, as in the case with the space radiator arrangement. Without the radiator, the specific impulse is constant and the main lower limitation of thrust is associated with a weight characteristic, namely, specific weight (see Figure 5). This is illustrated in Figure 7, which is a continuation of Figure 5 in the direction of higher values of thrust since, as mentioned before, the reactor weight is virtually independent of thrust.

The arrow in Figure 7 indicates the point at which the specific weight is equal to the corresponding parameter of a chemical rocket engine. This point is at a thrust of $10^{7} \mathrm{lbf}$; i.e., with respect to weight characteristics, the GCNTP engine is substantially a high thrust engine.

The foregoing considerations for both the space radiator design and the regenerative cooling approach make it possible to identify three basic types of GCNTP engine, the parameters of which are presented in Table 1. 


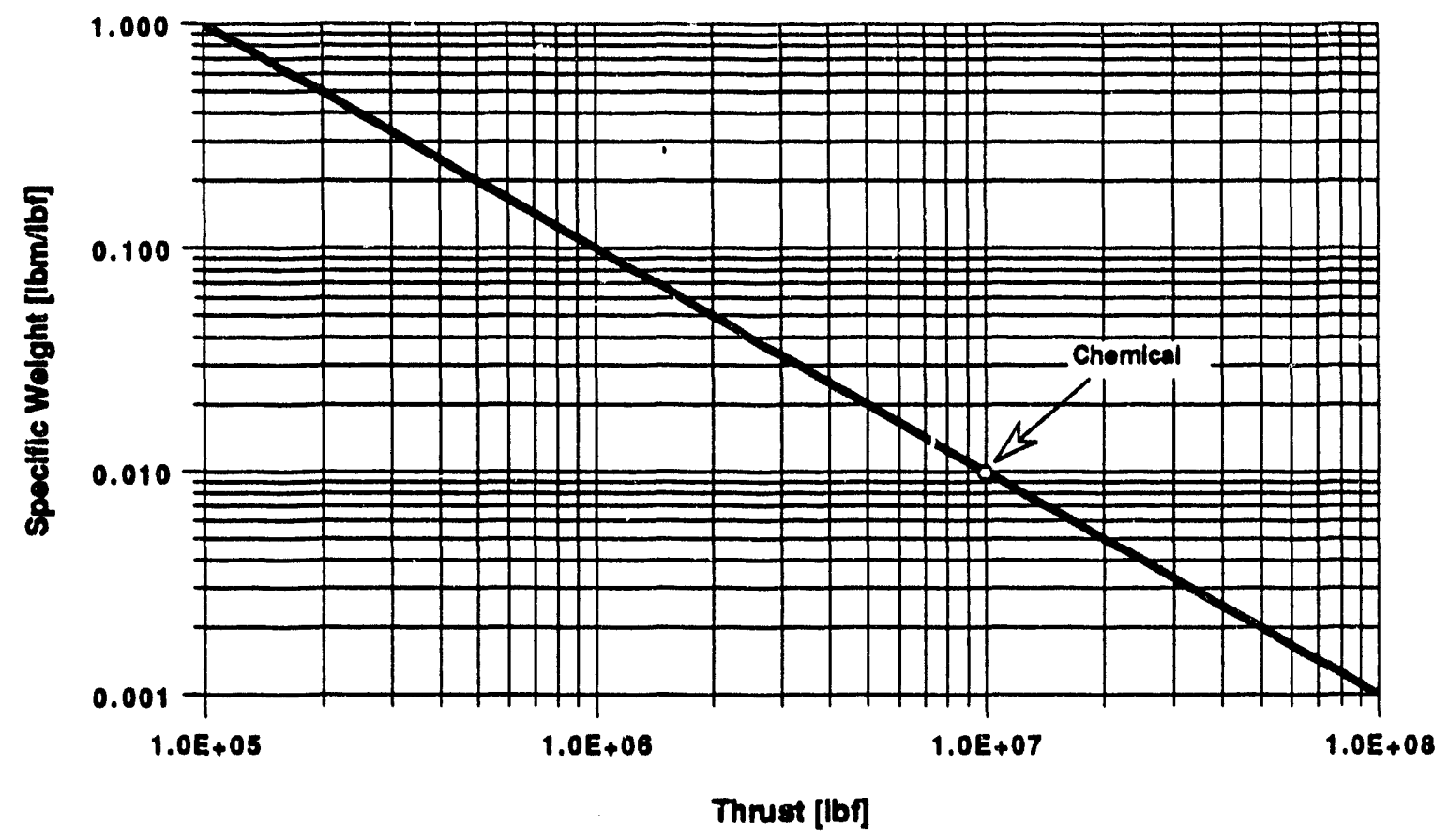

Figure 7. Dependence of Specific Weight on Thrust for the Gas Core Rocket Engine with Regenerative Cooling. 


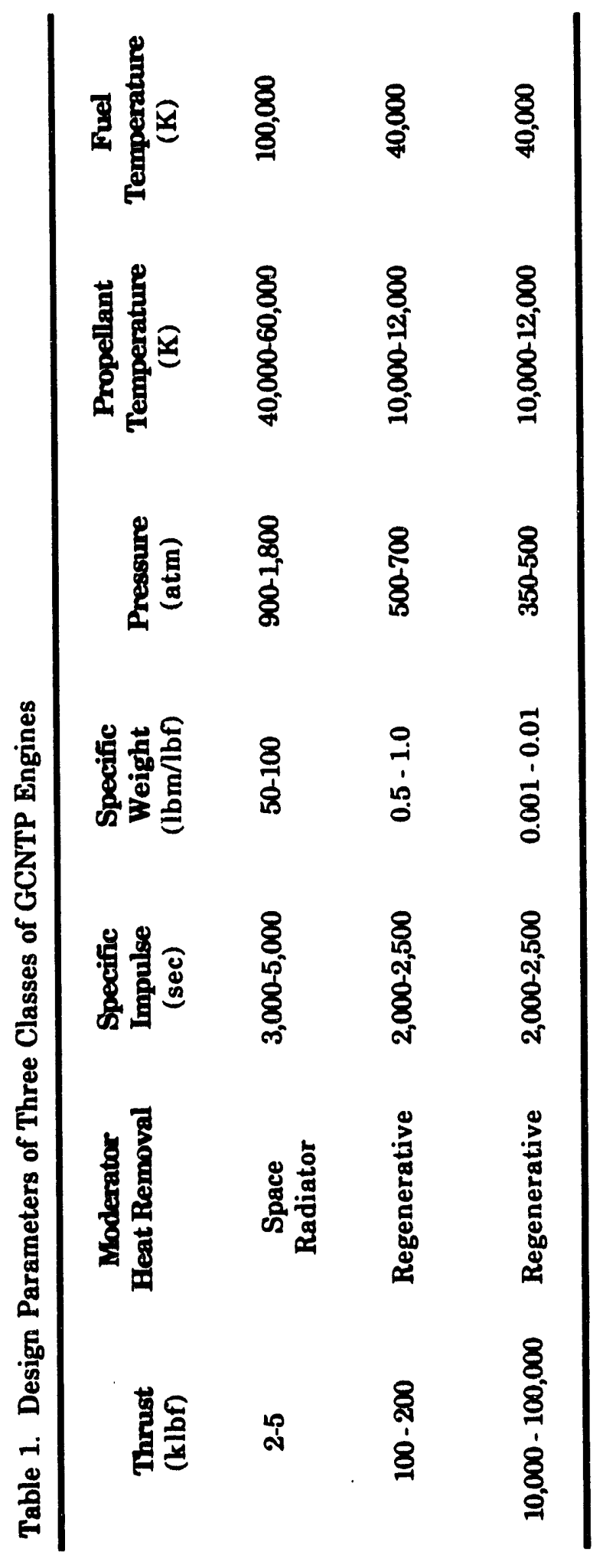




\section{OVERVIEW OF BASIC PROBLEMS}

\subsection{Entrainment of Fissionable Material}

There are several important aspects to the problem of confinement of the fissionable material in the reactor cavity. The mixing of uranium with hydrogen changes the molecular weight and, therefore, the dynamic characteristics of the propellant. The molecular weight of uranium is so great that even slight entrainment by the propellant can significantly reduce the specific impulse. Figure 8 presents the dependence of the ratio, $I_{s p} m i x / I_{s p}$, on the mass fraction of uranium in the mixture, $g_{U}$, with hydrogen as the propellant. As can be seen from the curve, at $g_{U}=10^{-1}$ the loss of specific impulse due to uranium contamination is $5 \%$, improving to $0.5 \%$ at $g_{U}=10^{-2}$.

A second consideration related to the entrainment of uranium is the effect on reactor control. Indeed, the average lifetime of various groups of delayed neutrons varies from $0.62-80.2$ seconds, and the groups with greater time make the main contribution to the reactor period. Thus, to ensure reactor control the uranium fuel residence time should not be less than several dozen seconds, which corresponds to a flow rate of about $2 \mathrm{lbm} / \mathrm{sec}$. For low thrust engines with propellant flow rate less than $10 \mathrm{lbm} / \mathrm{sec}$, uranium entrainment should not adversely affect reactor control. With higher thrust, and hence higher propellant flow rate, uranium entrainment may create a control problem when the propellant flow rate is greater than about $200 \mathrm{lbm} / \mathrm{sec}$.

It may be possible to satisfy the necessary retention condition by capturing the entrained uranium before it exits the cavity, condensing it, and returning it to the core zone. The results of approximate calculations are presented in Figure 9 in the form of a dependence of the portion of heat removed during cooling and condensation of the uranium, $\Psi_{U}$, on the thrust and the time the uranium remains in the core zone. This portion of the heat should be added to the portion of the heat released in the moderator and removed by the space radiator or the propellant as previously discussed.

It can be seen from Figure 9 that the portion of heat carried by the uranium increases sharply with decreasing thrust and decreasing time of uranium residence in the cavity. As the thrust increases and the residence time approaches ten seconds, this scenario with uranium capture and condensation becomes acceptable from the point of view of the heat balance and the limiting specific impulse.

Numerous calculations for different designs indicate that the Reynolds number for the propellant in the cavity is of the order of $10^{5}-10^{8}$; i.e., the flow is clearly turbulent. The entrained uranium mass flow rate (hence, uranium loss) in this case would be of the order of one to three times the hydrogen mass flow rate, which is absolutely unacceptable from the point of view of any of the aforementioned conditions, as well as cost. Moreover, the situation is not salvaged by uranium capture and condensation. Thus, it can be concluded that retention of gaseous uranium 


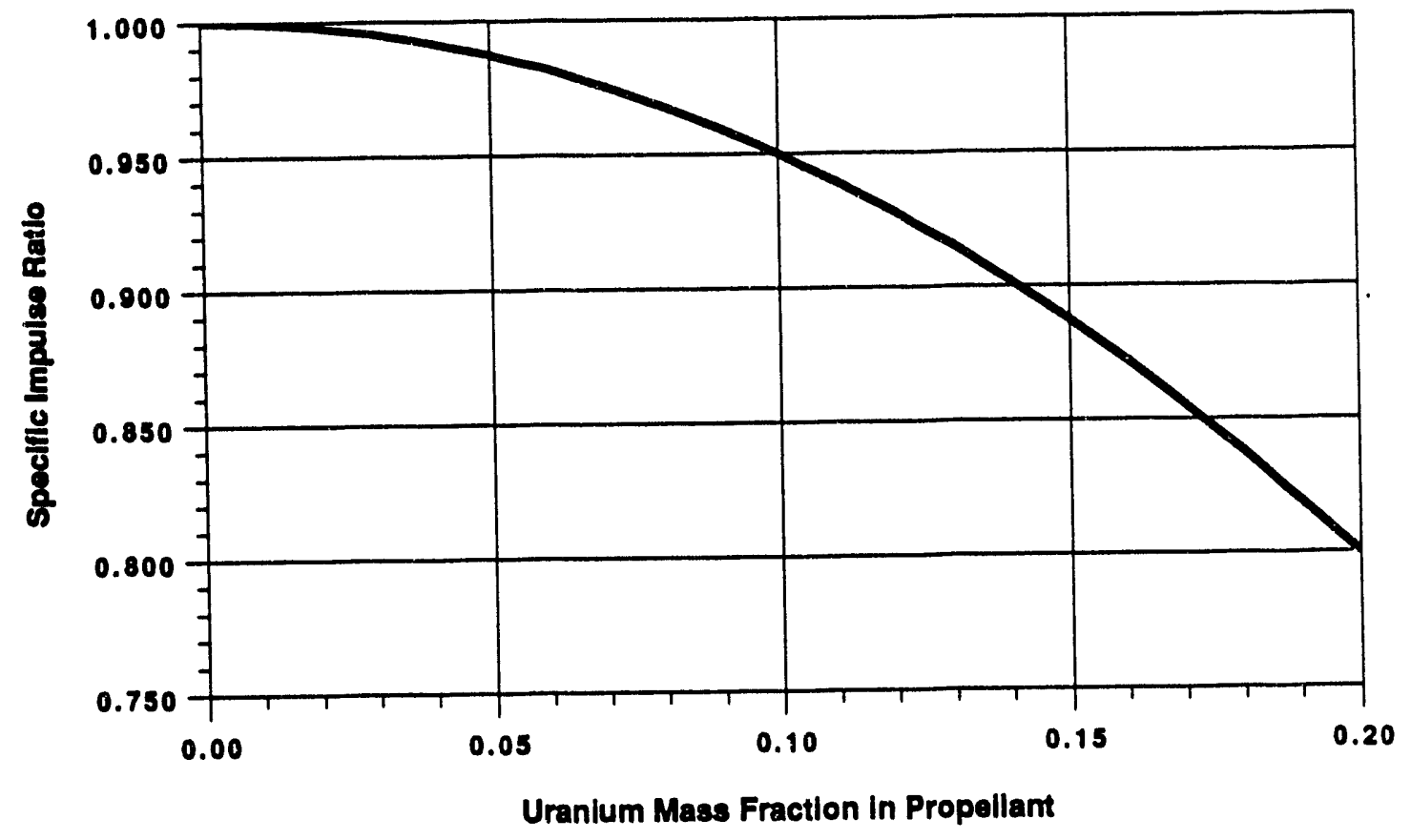

Figure 8. Reduction of Specific Impulse in a Gas Core Rocket Engine with Entrained Uranium. 


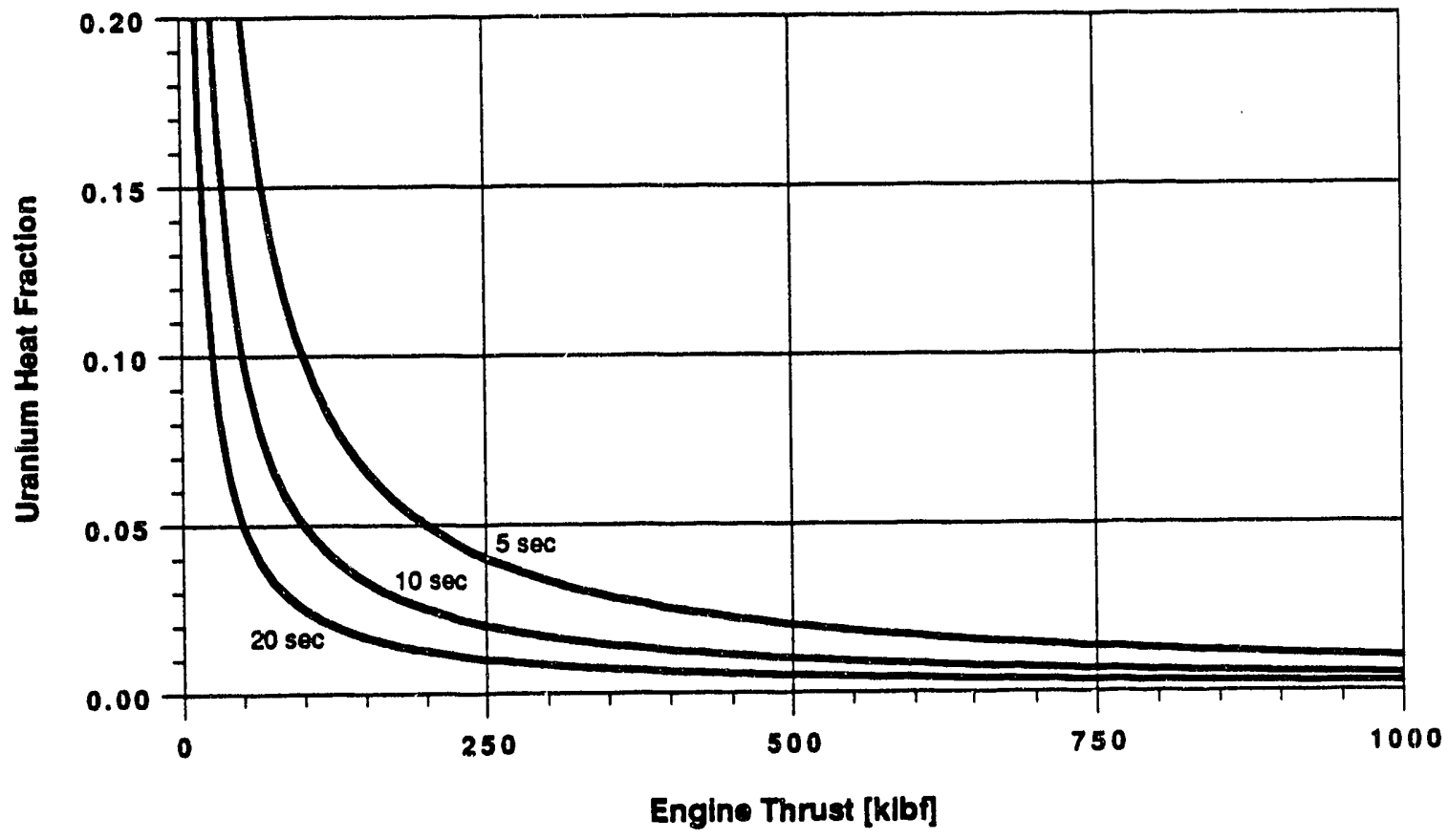

Figure 9. Dependence of Heat Removed by Entrained Uranium on Uranium Retention Time and Thrust. 
in the cavity requires laminar or nearly laminar flow of the propellant past the fissionable material.

Another important factor that destabilizes the zone of gaseous uranium is the force associated with the rocket's acceleration. Since the density of the gaseous uranium is some ten times greater than that of the hydrogen, the heavy uranium zone tends to fall out of the cavity through the nozzle. The velocity head of the propellant is great in comparison with the hydrostatic head of the column of gaseous uranium. However, it was found impossible to shape the duct to generate an "upward" longitudinal force that would keep the uranium from falling. For that reason, it is envisaged to install a support plate at the bottom end to prevent longitudinal movement of the uranium towards the nozzle at significant rocket accelerations and force it to flow laterally, which can be significantly retarded by means of a magnetic field as discussed in Section 7.5.

Estimates of uranium entrainment caused by various factors, including minimal laminar mixing, were made for different core zone designs. These estimates, which approximate the variability of the physical parameters of gas in the mixing layer, are presented in Figure 10 in the form of a dependence of the rate of flow of uranium, $m_{f} U$, on the Reynolds number, Re. As can be seen, uranium entrainment increases with Reynolds number, which is why one of the proposed ways of reducing entrainment was to create a reduced-velocity zone near the boundary between the propellant and the fissionable material.

\subsection{Requirements for Reactor Criticality}

Reactor physics calculations were carried out in which characteristic temperatures of the uranium, propellant, moderator, and construction materials were taken into account and varied. These calculations were performed along with fluid flow and heat calculations for design sensitivity. As a result, typical reactor dimensions, materials and uranium loading rates were determined.

Typical results from these studies were the following:

$\begin{array}{ll}\text { Cavity diameter } & 3-5 \mathrm{ft} \\ \text { Cavity length } & 5-8 \mathrm{ft} \\ \text { Moderator } & \text { Graphite } \\ \text { Reflector } & \text { Beryllium oxide } \\ \text { Structural materials } & \text { Oxides of titanium, zirconium and aluminum } \\ \text { Reactor mass } & 80,000-100,000 \mathrm{lbm} \\ 235 \mathrm{U} \text { charge } & 65-90 \mathrm{lbm}\end{array}$

In an alternate arrangement with modular fuel "cells" the critical loading was not changed significantly, since the increase in the amount of structural materials was offset by better moderator geometry. 


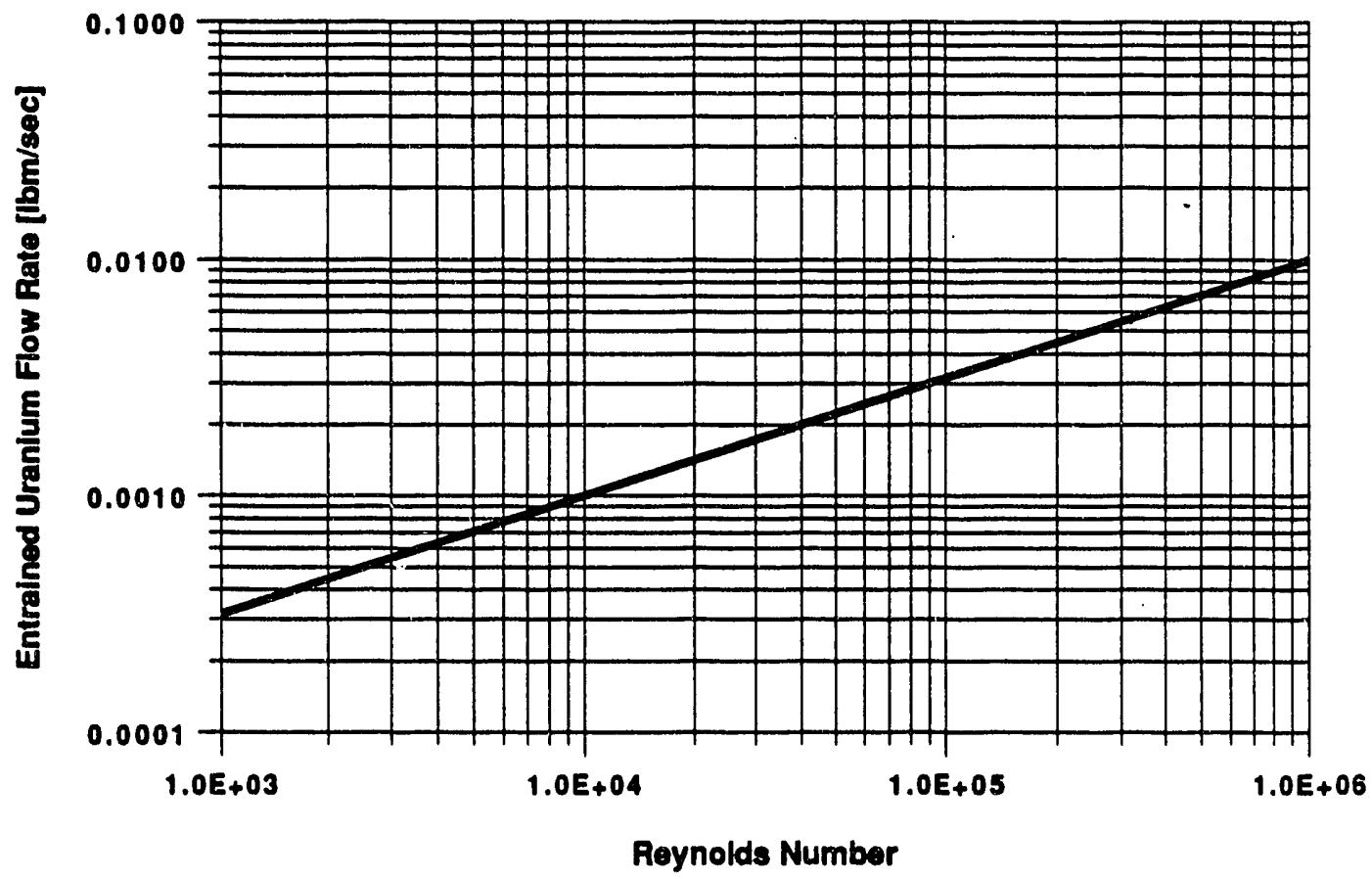

Figure 10. Dependence of Uranium Entrainment on Reynolds Number. 


\subsection{Reactor Control and Engine Startup}

The problems of starting the reactor, bringing it up to power, and achieving stable hydrodynamic and thermal conditions in the core were not studied in detail. Various theoretical calculations were performed, but there was no experimental work. Two ideas were proposed: placement of the startup charge in the moderator channels with subsequent injection as gaseous uranium into the core cavity; and placement of gas-actuated boron control rods in special channels with short characteristic actuation time.

\subsection{Properties of Dense Plasmas}

The study of the properties of dense (non-ideal) plasma is required to determine the thermodynamic properties and heat and mass transfer characteristics of gaseous uranium at high temperatures and pressures. Theoretical and experimental studies were carried out using several types of laboratory installations: shock tubes, arcs formed during static and impulsive discharges, and exploding wires. The range of parameters at which the tests were conducted differed significantly from operating parameters, so the corresponding theoretical estimates for prototypical reactor conditions were no more than a rough extrapolation.

It should be noted that the accuracy of determining basic parameters varied widely. In particular, the temperature of gaseous uranium was determined more precisely, since radiant heat transfer depends greatly on temperature. On the other hand, the accuracy of determining pressure was poor due to only an approximate knowledge of the equations of state for uranium plasma. In spite of the fact that methods for computing stationary processes in the core zone of a GCNTP reactor had been developed and employed extensively in designing conceptual variants, the imprecise knowledge of the physical parameters applied to those calculations makes it possible to use them only as estimates in the first approximation, not for engineering purposes.

\subsection{Radiant Energy Transfer}

The problem of radiant energy transfer is fundamental for two reasons: heating of the propellant and protection of the cavity wall. Furthermore, for medium and low thrust engines heat loss to the wall can lead to a substantial reduction in specific impulse, because it leads to an increase in the portion of heat removed by the propellant before it enters the cavity.

Calculations show that the radiant flux has a linear dependence on thrust at a constant specific impulse. For example, consider an $I_{s p}$ of 2,000 sec. For a thrust of $2.2 \times 10^{5} \mathrm{lbf}$, the radiant flux is $1.1 \times 10^{9} \mathrm{Btu} / \mathrm{ft}^{2} \mathrm{hr}$; and at $2.2 \times 10^{7} \mathrm{lbf}$, it is $1.1 \times 10^{11} \mathrm{Btu} / \mathrm{ft}^{2} \mathrm{hr}$. For medium and large thrusts the fraction of heat radiated to the wall (from the point of view of thermal protection) should not exceed $10^{-2}$ and $10^{-4}$, respectively, of the total heat generated. From the point of view of the 
specific impulse, the portion of heat radiated to the wall should not exceed several percent. All of this imposes very rigid requirements on the absorption characteristics of the propellant.

Since the hydrogen at the cavity inlet (temperature $\leq 2,800 \mathrm{~K}$ ) is virtually transparent to thermal radiation, it is commonly proposed to darken it with atomized particles of a refractory metal. This allows heating to temperatures approaching $5,500 \mathrm{~K}$. Furthermore, a small addition of an alkali metal would lead to the formation of negative hydrogen ions making a significant contribution to absorption and facilitating further heating of the hydrogen to operating temperatures above 5,500 K. Experiments and calculations show that in this case it is possible to use an optically dense approximation of radiant heat conductivity, which considerably facilitates the development of computational methods.

It should be noted that near the wall, where the temperature is below $5,500 \mathrm{~K}$, the only mechanism of heat transfer remains absorption on particles suspended in the gas. Thus, the entire flow field of the propellant is divided by the approximately $5,500 \mathrm{~K}$ isotherm into two zones with their respective radiation absorption mechanisms, and in the cavity outflow the line passes in immediate proximity to the wall. It is important to stress that this picture of radiation absorption by the propellant is the primary basis of the reactor operation, and no other alternatives were proposed.

\subsection{Thermal Protection of Cavity and Nozzle Walls}

It was shown before that the operating condition in the cavity is to a significant degree determined by the absorption capability of the propellant. The propellant is blackened by special additives to the extent that it becomes possible to apply approximations of radiant thermal conductivity for both the high- and low-temperature zones. Numerous computations have shown that the radiant heat conductivity near the wall, which is strongly dependent on temperature, is commensurate with turbulent heat conductivity. In the outlet cross-section of the core, where the heat flow to the wall is highest, it can reach about $3 \times 10^{7} \mathrm{Btu} / \mathrm{ft}^{2} \mathrm{hr}$. A much more intense zone is in the neighborhood of the nozzle throat, where the main transfer mechanism is turbulent and convective heat transfer can reach $10^{10} \mathrm{Btu} / \mathrm{ft}^{2} \mathrm{hr}$.

Thermal protection is also required for the prechamber walls of the multi-cavity design. The flow of gaseous fissionable material must be directed away from the wall, otherwise the uranium will condense, since its boiling temperature at the characteristic pressures of the gas reactor is upwards of $6,000 \mathrm{~K}$, whereas the wall temperature would hardly exceed $2,500 \mathrm{~K}$. The formation of a uranium film would lead to high heat flows which would be impossible to remove. Thus, injection into the hydrogen boundary layer is required for the partial pressure of uranium at the wall to be lower than the vapor pressure at the wall temperature. Estimates show that the volume fraction of uranium should be $10^{-2}-10^{-3}$ near the wall. 
To protect the wall of the three most heat-intensive parts of the engine, the heat flux in a turbulent boundary layer must be reduced by several orders of magnitude. Extensive experimental and theoretical studies showed that this problem could be resolved most economically by injecting propellant through a porous wall. The need to reduce the parameters at the wall by several orders of magnitude in comparison with conditions without injection called for studies in "strong injection" conditions. It was shown that at Reynolds numbers typical of flows in the cavity and nozzle, the required ratio of the rate of injection to the velocity of the main stream was approximately $0.005-0.02$.

It should be noted that at sufficiently low values of thrust, the amount of coolant needed for injection and external cooling of the cavity and nozzle walls noticeably affects the specific impulse and leads to an appreciable reduction in the limiting specific impulse.

\subsection{Acoustic Instability}

Fissioning uranium plasma can experience thermoacoustic oscillations associated with the volume distribution of generated heat. NII TP personnel developed an energy method theory for this, making it possible to attain sufficient degrees of stability depending on the intensity of heat generation, heat transfer, phase relationships between pressure and heat generation pulses, and acoustic conditions at the volume boundary.

The energy method theory was used to demonstrate the strong stabilizing effect of a magnetic field and obtain the dependence of the field intensity on controlling factors. However, the strong dependence of stability conditions on gas boundary conditions and physical parameters makes it possible to regard the quantitative results only as rough orders of magnitude. Although a theoretical description of the method has been developed, no computational methods have been developed which could select the parameters that ensure the stability of the process. 


\section{CONFINEMENT OF GASEOUS FISSIONABLE MATERIAL}

Confinement of gaseous fissionable material in the reactor cavity is one of the key problems of the GCNTP engine. Confinement means ensuring that the critical loading is maintained while the loss of material does not exceed certain limits which depend on operating conditions such as the duration of engine firing and thrust. Compromise solutions are possible, but the loss must be as small as possible. Given the hydrodynamic conditions in the cavity, confinement is an extremely difficult task.

\subsection{Cavity Flow Characteristics}

First, consider the steady flow in the single cavity engine shown in Figure 3. The overall picture is an axially symmetric flow of propellant past a slowly moving "stagnation" zone of fissionable material and passing through a narrow annular channel formed by the surface of the fissionable material and the wall of the cavity. The propellant is fed to the cavity through an intake honeycomb which eliminates turbulent disturbances and forms a velocity profile increasing from a small value at the gaseous uranium interface to a higher value near the wall. Further movement of the hydrogen along the channel is determined by the thermal input and the magnetic field directed along the cavity axis.

The propellant mean temperature increases by a factor of 4 to 4.5 , leading to a corresponding increase in the mean velocity. The radial gradient of the longitudinal velocity varies with the motion of the propellant. The hotter layers of hydrogen adjoining on the uranium zone accelerate, leading to a significant deviation of the velocity profile from the one established by the inlet device and to the appearance of an associated radial component of the velocity. The radial component, in turn, interacts with the longitudinal magnetic field, which retards this motion. Detailed computations confirm that as the hydrogen moves along the cavity the velocity distribution that formed at the intake changes into a much more complex two-dimensional flow.

Development of the hydrodynamic flow pattern depends strongly on the variability of the physical properties of the moving gas. Radiant heat transfer largely determines gas temperature and density. Electrical conductivity, which varies with gas temperature, also greatly affects the hydrodynamics. Near the uranium interface the temperature is high and the magnetic field has a strong stabilizing effect, minimizing any lateral flows. On the other hand, near the wall, or more precisely, between the wall and the approximately $5,500 \mathrm{~K}$ isotherm the electrical conductivity is close to zero and the magnetic field has no effect. Here the flow is shaped by the pressure gradient, which is weaker in this zone because of the relatively higher density.

The velocity of the uranium zone is low, determined by the need to compensate for the loss of fissionable material due to entrainment and rocket acceleration. A protective layer of alkali metal comes into contact with the hydrogen flow, and uranium diffuses into this layer. Effective 
formation and preservation of the protective layer is possible only in the presence of a magnetic field; the stabilizing effect on the layer is great due to the metal's high electrical conductivity.

In the multi-cavity arrangement with circulation of the fissionable material, rocket acceleration helps uranium to flow along the cavity, and the stream enters the intake. The required velocity of the uranium flow at the cavity intake is fed into the prechamber.

In the single-cavity arrangement the gaseous uranium zone "rests" on the end plate. Longitudinal acceleration of the rocket produces a pressure gradient which causes the uranium to move, together with the alkali metal protective layer, in a radial direction. This motion is opposed by the magnetic field, and the radial velocity, which determines the loss of uranium, is established as a balance of acceleration and Lorentz forces.

The mean velocity of the propellant is affected by different factors acting in opposite directions. The main factors are the need to heat the propellant but without too great a heat flow to the wall. Furthermore, the velocity should not be too high, so that magnetohydrodynamic stabilization of the flow can be effective. The width of the annular cross-section through which the hydrogen moves cannot be too great because of considerations of neutron physics, however, it must be large enough to prevent the boundary layers from affecting the main flow too greatly.

Many variants were numerically analyzed. They revealed that the specific geometry of the flow section of the cavity and gaseous uranium zone was not a significant factor and that the important parameters were the radiant heat transfer and electrical conductivity of the fluids, as well as the velocity of the propellant. The calculations suggested the ranges of the hydrodynamic parameters presented in Table 2. 
Table 2. Range of Reactor Characteristics and Hydrodynamic Parameters in the Single-Cavity Gas Core Rocket Engine

Reactor Characteristics

Cavity pressure

Mean velocity of propellant at intake section

Mean density of propellant at intake section

Mean density of propellant at outlet section

Width of annular cross-section of propellant channel

Cavity length

Mean uranium density

Uranium zone diameter
Range

$350-1,000 \mathrm{~atm}$

$100-1,150 \mathrm{ft} / \mathrm{sec}$

$0.3-0.5 \mathrm{lbm} / \mathrm{ft}^{3}$

$0.1-0.13 \mathrm{lbm} / \mathrm{ft}^{3}$

$0.25-0.5 \mathrm{ft}$

$3.3-5 \mathrm{ft}$

$0.6-0.9 \mathrm{lbm} / \mathrm{ft}^{3}$

2.6-3.6 ft

\section{Dimensionless Hydrodynamic Parameters of Propellant}

Reynolds number

Magnetic Reynolds number

Prandtl number based on radiant difiusion

Hartmann number

Stuart number
$10^{5}-10^{7}$

$10^{-3} \cdot 10^{-5}$

$10^{-5}-10^{-6}$

$3 \times 10^{3}-10^{5}$

$10^{2}-10^{3}$

\subsection{Delaying Transition to Turbulence}

When no special measures are taken, the velocity profile of submerged streams and wakes can abruptly change. The transition to turbulent flow is very rapid and is stimulated by the presence of initial turbulent disturbances in the streams forming the jet flow. A reduction in the turbulent intensity of the main stream increases the transition Reynolds number, particularly in the boundary layer. Furthermore, beyond the point where the flow becomes unsteady, a certain distance along the stream is required for long-wave disturbances to develop into turbulence. The flow at the boundary between gaseous uranium and propellant in the cavity is approximately a jet flow, because the cavity wall is sufficiently far away.

Extensive experimental studies of jet flows were carried out at NII TP during which the effects of initial conditions on the transition to turbulence were studied. Two factors were important as initial conditions: the degree of turbulence and the velocity distribution in the initial crosssection. The initial velocity profile was developed with the help of a honeycomb with variable channel resistance. Cell size, edge thickness, Reynolds numbers, and gas density were varied. Typical results are presented in Figure 11. 

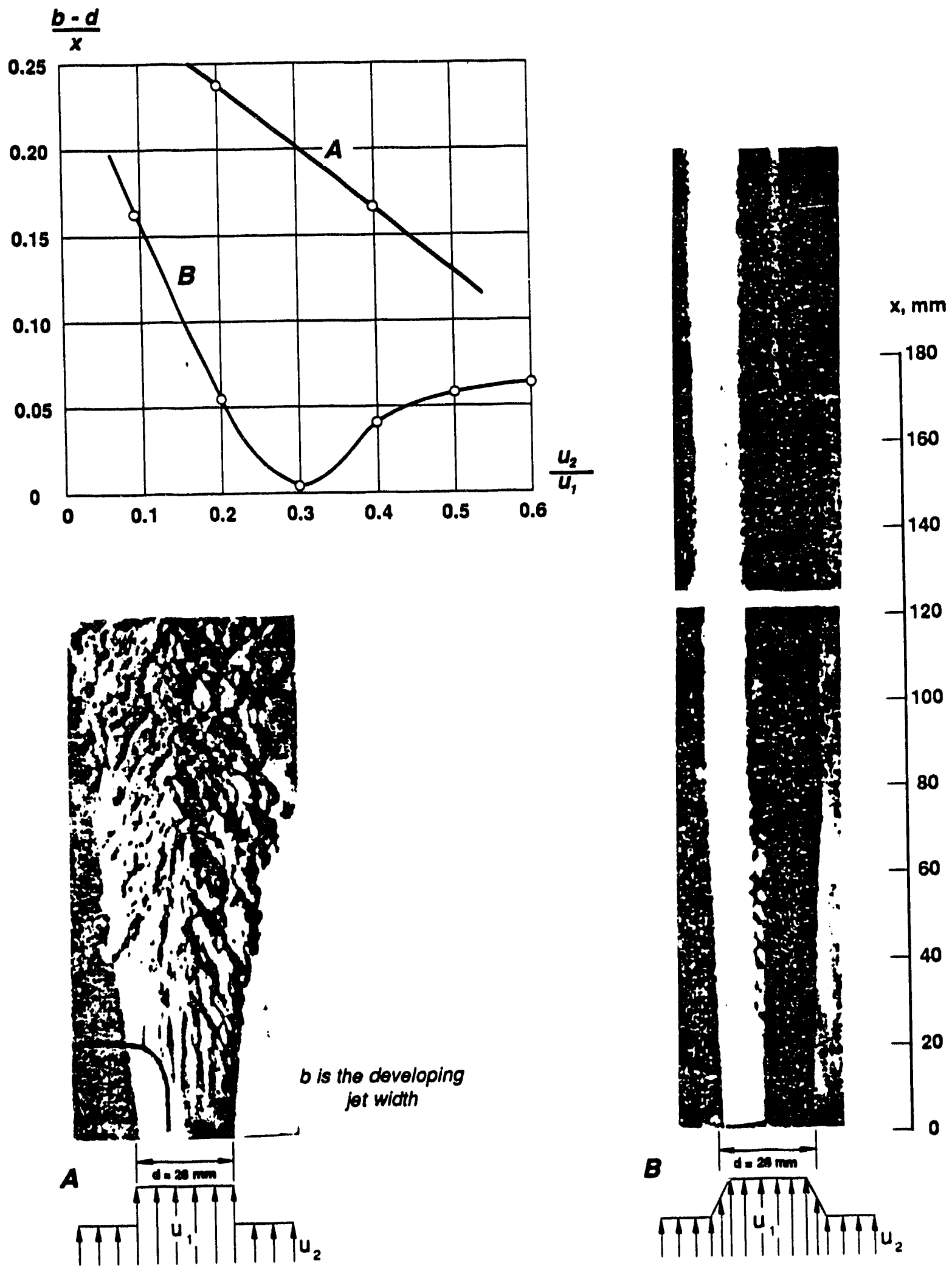

Figure 11. Helium Jet in Coaxial Air Stream. Sources: O. I. Navoznov and A. A. Pavelyev, Izv. AN SSSR, MZhG, 6, 1969, and O. I. Navoznov and A. A. Pavelyev, Izv. AN SSSR, MZhG, 4, 1972. 
It can be seen that in the case of a smooth initial velocity profile the jet remains smooth, there are no turbulence pulses, and flow remains laminar. Tests showed that by judicious selection of honeycomb parameters it was possible to maintain laminar flow over a considerable distance up to ten diameters of the initial variable velocity layer. This is an important qualitative result as applied to the problem of confining the fissionable material in the gas core reactor.

Unfortunately, the experiments showed a strong dependence on the Reynolds number, which was 4 to 5 orders of magnitude below reactor conditions. Furthermore, the high heat transfer rate in the cavity leads to a rapid deviation of the profile from its initial value. Certain restrictions on the extent of the laminar flow may also be imposed by the development of natural convection which depends on the rocket acceleration.

Overall, it was determined that the use of special intake honeycombs can resolve the problem of flow stabilization in the region where the propellant has not yet heated to the temperature where electrical conductivity becomes sufficient for a magnetic field to effectively stabilize the flow.

\subsection{Suppression of Turbulence}

A magnetic field strongly affects the flow of an electrically conductive fluid. To avoid distortion of the main stream in the cavity, the field must be directed along the streamlines of the propellant. At the same time the magnetic field always affects the turbulent flow, since turbulence is basically three-dimensional flow, i.e., there is always a velocity component perpendicular to the magnetic field vector.

An important magnetohydrodynamic parameter is the magnetic Reynolds number, which characterizes the effect of motion on magnetic field strength. In the cavity, the magnetic Reynolds number is substantially less than unity, being in the range, $R e_{m}=10^{-3}-10^{-5}$. The magnetic field in the cavity is produced externa.iy by a solenoid magnet. The principal qualitative feature which characterizes MHD turbulence at low $\boldsymbol{R e}_{\boldsymbol{m}}$ is that interaction with the magnetic field leads only to the appearance of a Joule dissipation mechanism.

When NII TP began work on a gas core reactor there were not many experimental studies indicating the suppressive effect of a magnetic field on turbulence. Such studies were launched on an extensive scale at the Institute.

Experimental data on the attenuation of uniform turbulence of liquid metal passing through a grate are presented in Figure 12a as the time dependence of a component perpendicular to the magnetic field. It can be seen that the attenuation of turbulence occurs monotonically and very quickly over the entire range of studied values of dimensionless time.

The characteristic MHD time is defined by the relationship: 


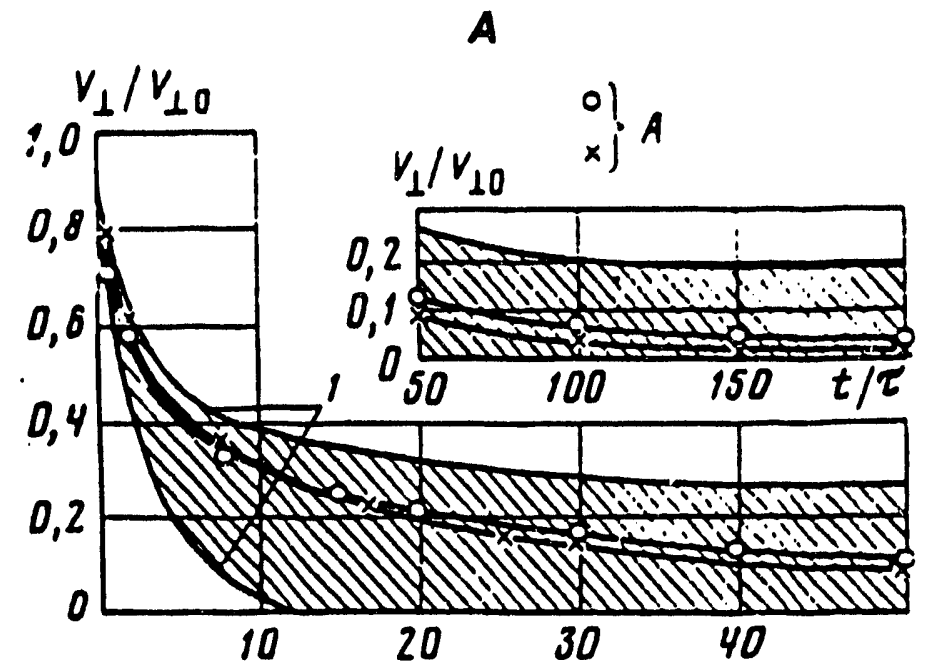

Graph A: Attenuation of uniform turbulence: where $v$ is the turbulent velocity component perpendicular to the direction of the magnetic field Shaded region: Experimental data from [1]

Curve1: Computation of limiting cases according to [2]

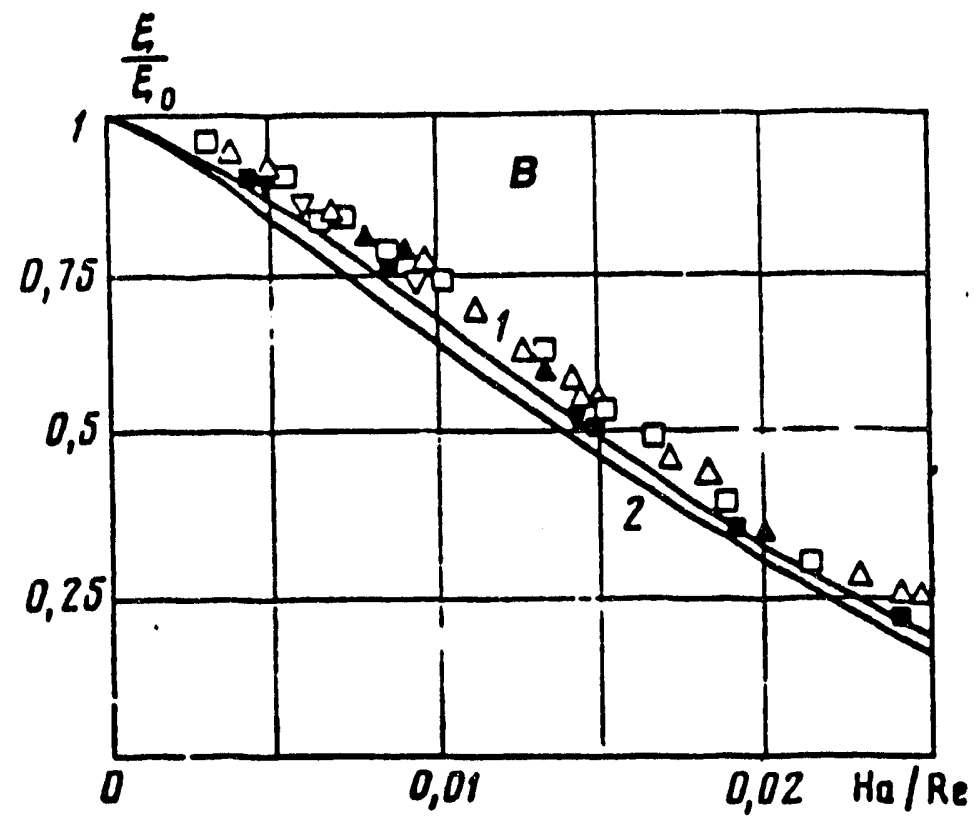

Graph B: Drag factor for liquid metal flow through a pipe in a longitudinal magnetic field. Experimental data from [3].

Curves1 and 2: Computation according to [2] for limiting parameters in experiments.

[1] A. V. Volkov, MG, 4, 1975.

[2] V. M. levlev, Turbulent Motion of High-Temperature Continuous Media, Nauka, 1975.

[3] V. B. Levin and I. A. Chinenkov, MG, 3, 1970.

Figure 12. Suppressing Effect of a Magnetic Field on Turbulent Flow. 


$$
\tau=\frac{c^{2} \rho}{\sigma H^{2}}
$$

This quantity lies in the range of $10^{-4}-10^{-6}$ seconds for conditions in the cavity. If $t$ is the time of interaction of the magnetic field on the flowing fluid, then the ratio $t / \tau \geq 200$ is required for the virtually complete suppression of turbulence. This is achieved over a length of several centimeters, if computations are based on the typical mean velocity. A more accurate calculation would be according to the velocity of the propellant close to the uranium, which would yield an even smaller value.

Figure 12b presents experimental data on the reduction of the drag factor during motion of a liquid metal through a long round pipe in a longitudinal magnetic field. The experimental points form a simple dependence on the ratio of the Hartmann number to the Reynolds number, Ha/Re. Experimental data indicate the drag factor decreases monotonically down to the value 0.025 , which is in the range of characteristic values of this ratio in the cavity.

An example of experimental data on the effect of a longitudinal magnetic field on jet flows is presented in Figure 13. Figure 13a shows the contraction of the mixing zone width in the magnetic field. As the magnetic field increases the growth of the mixing zone along the stream becomes smaller because of reduced turbulent transfer due to suppression of turbulence pulses. There is a similar explanation for the narrowing of the concentration profiles for a plasma filament in axial flow, as shown by the experimental data in Figure 13b.

These data indicate that suppression of turbulence by a magnetic field, in the sense of the reduction of mixing, has been proven experimentally. In addition, there are sufficiently detailed theoretical methods making design calculations possible. ${ }^{5}$

In the case of flow in a cavity, uniform turbulence can be suppressed rapidly by a magnetic field in the absence of a turbulence producing mechanism. Of course, turbulence production is present in gas core flows with internal fluid shear layers. Further, at Reynolds numbers characteristic of the gas core, the mechanism is very strong. As is shown in Figure 12b, even at very high values of the $\mathrm{Ha} / \mathrm{Re}$ parameter, the turbulent drag coefficient decreases by a factor of less than 4 in comparison with the absence of a magnetic field.

During engine operation turbulent transfer exceeds molecular transfer by 2 to 4 orders of magnitude. Solving the problem of reducing mixing at the uranium zone boundary to the molecular level involves either choosing a sufficiently strong magnetic field to suppress turbulence in a flow with a mean velocity gradient, or by creating a flow near the uranium interface that would have a small or zero mean velocity gradient. Then the required magnetic field would be much smaller. With regard to the thermal protection of the core components, the temperature near the wall is low enough so that the electrical conductivity is effectively zero, and the magnetic field has no influence in this region. 

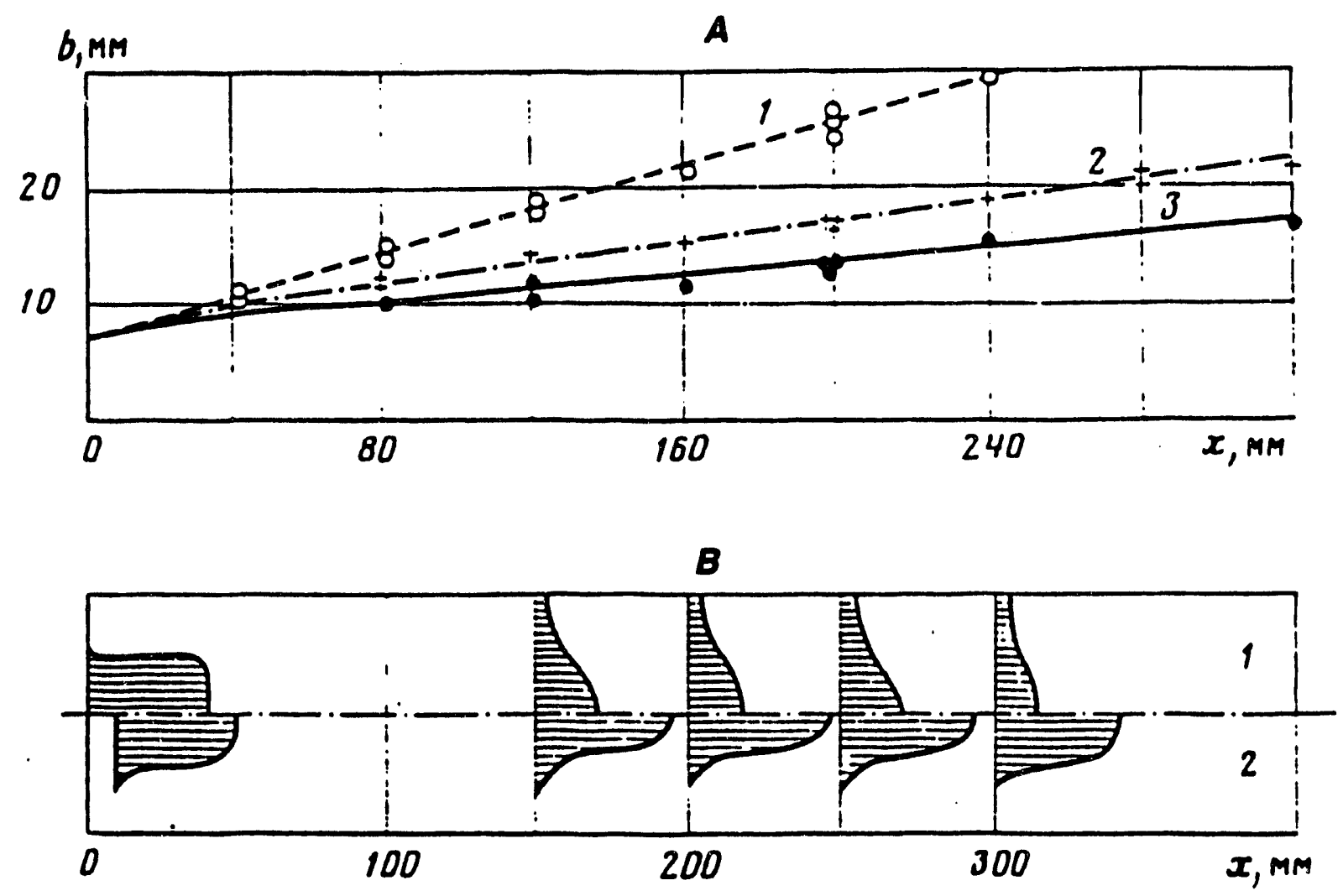

Graph A : Example of dependence of displacement zone width, b, on distance, $x$, for a liquid metal flow filament from [1] and [2]

Cunv 1: $\mathrm{H}=0 \mathrm{Oe}$

Curve 2: $H=2,400 \mathrm{Oe}$

Curve 3: $H=5,000 \mathrm{Oe}$

Graph B: Concentration profiles for a flow filament and plasma in confluent flow

Curve 1: (above the dashed line) - In the absence of a magnetic field

Curve 2: (below the dashed line) - In the presence of a magnetic field

(the Stuart number for the filament mixing zone is close to 3 ).

[1] S. S. Freobrazhensky and I. A. Chinenkov, MG, 2, 1970.

(2) B. N. Baushev, Ye. Yu. Krasilnikov, V. G. Lushchik, and I. G. Panevin. Izv. AN SSSR, MZhG, 5, 1972.

Figure 13. Effect of a Longitudinal Magnetic Field on the Mixing Layer Growth in Jet Flows. 


\subsection{Increasing Laminar Flow Stability}

In fluid dynamics the problem of the stability of laminar flow with respect to infinitesimal disturbances is distinct from the transition to turbulence since it defines loss of stability as a point at which an unsteady mode appears and grows exponentially with time. A similar methodology was applied to the MHD case, the general conclusion being that a magnetic field enhances stability with respect to infinitesimal disturbances. Exceptions to this include the special case of twodimensional disturbances in a plane perpendicular to the magnetic field, as well as cases where the field significantly affects the mean velocity profile.

When applied to flow in the engine, the problem is posed differently: We need to determine the sufficient condition for the absence of turbulence, i.e., establish a criterion of stability with respect to finite (not infinitesimal) disturbances. The numerous and reliable experimental data on transition to turbulence during flow through a round pipe in a longitudinal magnetic field can be expressed to a high degree of accuracy by the equation:

$$
\frac{R e_{c r}}{R e_{c r}^{o}}=1+0.4 S
$$

At high values of the Stuart number we obtain a simple expression for the transition condition:

$$
\left(\frac{H a}{R e}\right)_{a r}=0.033
$$

The physical meaning of the linear dependence of the critical Reynolds number on the Stuart number is that this condition corresponds to the equal production and dissipation of disturbed motion. A similar condition was obtained theoretically for flow in a planar shear layer. ${ }^{6}$ Furthermore, theoretical and experimental conditions were obtained for cases of flows of entirely different configuration: annular Couette flow in a coplanar magnetic field with a rotating inner cylinder: $(H a / R e)_{c r}=0.015 ;^{7}$ and flow between coaxial cylindrical surfaces in longitudinal magnetic and radial electrical fields: $(H a / R e)_{c r}=0.038 .^{8}$ The higher number in the latter case is due to the nature of the flow itself. On the other hand, the low value of the criterion for a coplanar field is due to a change in stability and transition to less "dangerous" Taylor eddies.

On the whole, it can be said that the stability criteria with respect to finite disturbances, $(\mathrm{Ha} / \mathrm{Re})_{\mathrm{Cr}}$, is known to a satisfactory degree of accuracy. 


\subsection{Effects of Rocket Acceleration on Uranium I.oss}

The density of the fissionable material is some ten times greater than that of the propellant. Depending on the acceleration, a pressure gradient develops along the radius of the uranium zone, leading to radial displacement, since the end plate prevents axial displacement. Approximate determination of uranium loss due to rocket acceleration, taking into account the stabilizing effect of a longitudinal magnetic field, assumes that only the pressure of the uranium column and the Lorentz force are balanced. In that case we obtain the following expression for the fissionable material radial velocity versus distance from the upper cross section of the engine:

$v=\frac{c^{2} \rho \times a}{\delta \sigma H^{2}}$

The volume flow is obtained by integrating this expression over the entire length of the uranium zone. The results for typical parameters are presented in Figure 14 as a function of the time the uranium remains in the cavity, the acceleration, and the magnetic field. It can be seen that at the time required for laminar flow, approximately $10^{3} \mathrm{sec}$, the magnetic field intensity must be in the range of $1-10$ tesla.

In conclusion we note that the pressure drop associated with the acceleration of the propellant has a value commensurate with the hydrostatic pressure of the uranium column, thus the results of Figure 14 have a more general significance.

\subsection{Using a Magnetic Fleld to Stabilize Flow}

\section{Rotational Motion in the Mixing Zone}

The boundary of the fissionable material zone is a mixing zone of materials with different ionization potentials, so that the electron concentration varies with the radius. Given a longitudinal temperature gradient, this must lead to the appearance of thermoelectric current, the radial component of which interacts with the longitudinal magnetic field, producing rotational motion. A similar effect applied to turbulent motion was considered before. ${ }^{9}$ Rotation of the displacement zone can lead to the appearance of secondary currents and turbulence, especially since the density decreases towards the periphery.

There are no strict theoretical solutions or experimental data for such a complex flow and stability problem. However, qualitative and approximate quantitative analyses could be carried out on the basis of experimental data on the stability of rotational flows. Disregarding viscous forces, the rotational velocity can be determined by equating the thermoelectric and induced electrical fields: 


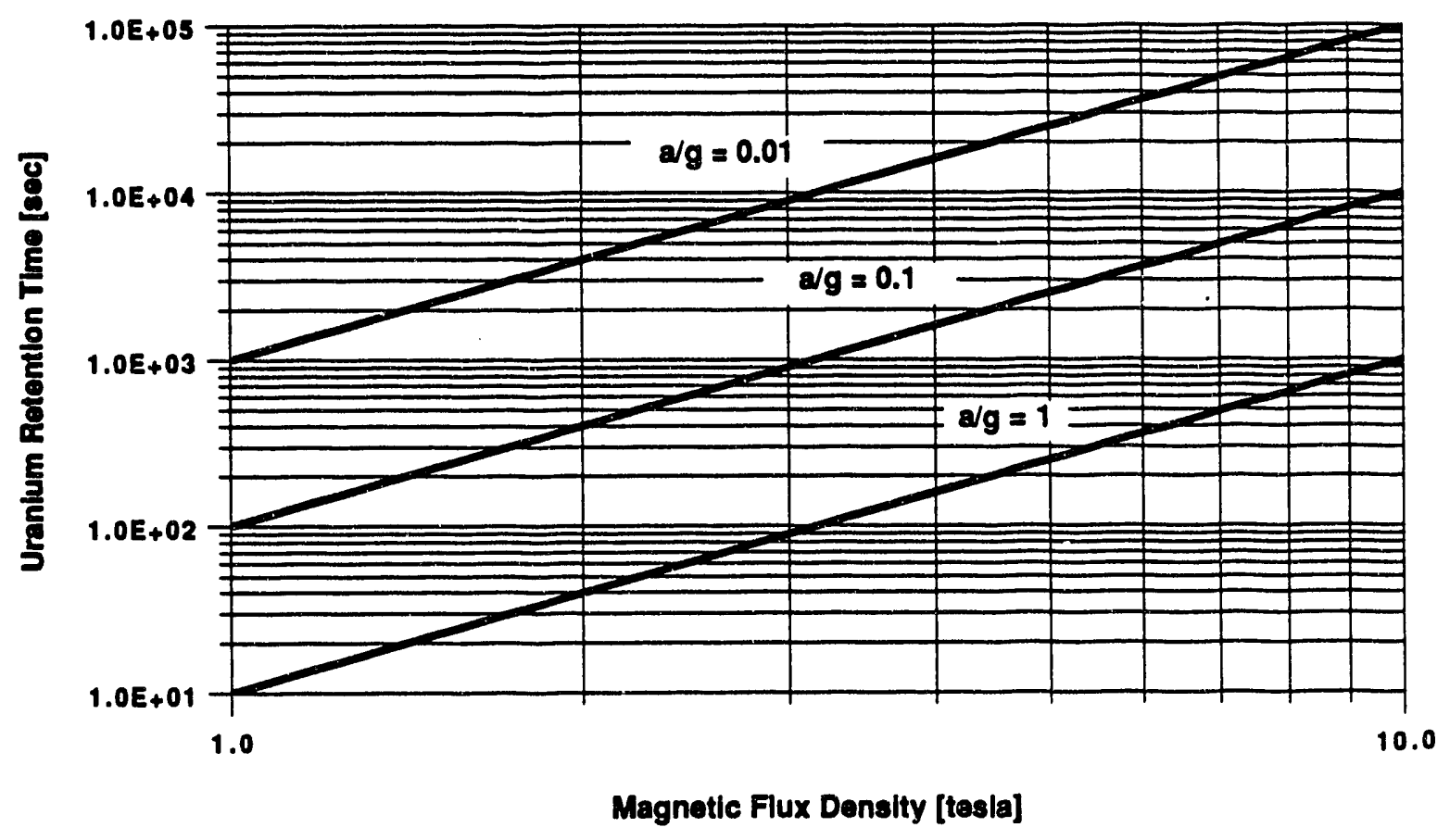

Figure 14. Dependence of Uranium Retention Time on Magnetic Flux Density and Rocket Acceleration. 
$\vec{E}_{T}=\frac{1}{c} \vec{v} \times \vec{H}$

where the thermoelectric field strength $E_{T}$ is determined according to the composition of the mixing zone and the longitudinal temperature gradient.

If we use the condition of stability of rotational flow in the form:

$\frac{H a}{R e}=$ constant

from these two equations we can determine the necessary magnetic field, which must satisfy the condition:

$\frac{H^{2} \sqrt{\sigma \mu}}{c^{2} \rho E_{T}}=$ constant

Estimates using this equation yield a value for the required magnetic field of 7-10 tesla.

Interaction of the Propellant Flow with the Magnetic Field

The magnetic lines of force are directed along the velocity vector in the cavity. However, downstream at the nozzle intake the streamlines of the propellant inevitably intersect the magnetic field intensity lines. Estimates indicate that the corresponding pressure difference can reach several pounds per square inch, which is commensurate with and even exceeds the velocity head of the propellant in the cavity. Since in a magnetic field the effect of various fluctuations in the velocity field extends upstream for a considerable distance, this effect may have a negative impact on the flow pattern in the cavity.

\subsection{Required Magnetic Field Intensity}

In Table 3 we present a summary of magnetic field intensities necessary to stabilize several different types of flow instabilities. These data were obtained on the basis of experimental results in model experiments and approximate computations that extrapolated them to flow in a gas core reactor cavity. 
Table 3. Magnetic Field Strengths Required to Stabilize Undesirable Flow Characteristics

Type of Instability

Acoustic instability

Hydrodynamic instability (turbulence suppression)

Longitudinal acceleration

Rotational instability
Required Magnetic Field

2-3 tesla

3-4 tesla

3-5 tesla

7-10 tesla 


\section{ISSUES IN SUBSEQUENT R\&D}

\subsection{Current Status}

The current state of development of the GCNTP should be considered from two points of view. First, the engine must be defined from the point of view of the performance necessary to meet space mission requirements, including engineering and technical issues that may arise during practical development of a space vehicle with such an engine. Secondly, a proof of principle must be conducted to demonstrate the practical feasibility of the concept.

These two problems are at entirely different stages of realization. The first can be considered as the more resolved, whereas the second problem has not yet passed an important stage essential to confirm its practical feasibility.

\section{Engine Design Parameters}

We can consider that the overall GCNTP parameters are, at this stage, known with general accuracy. They include the choice of propellant, cavity pressure, specific impulse, and the total weight. Then, given the necessary thrust, it is possible to design all engine systems which will make it possible to define the weight characteristics needed to carry out more accurate trajectory computations. In our work, such scoping computations were conducted on a continuous basis to take into account updated numbers, including the weight of the nuclear radiation shield.

Conceptual designs for all engine components were developed. It is important to stress that the main problems associated with a graphite moderator have been resolved to the same extent that they have been for a solid core nuclear engine, since the operating conditions of both these units are fairly similar.

On the whole it can be said that the available engine requirements and data are a good foundation for producing working designs of a specific engine, and for planning manufacturing and testing of a prototype.

\section{Proof of Principle}

From the point of view of the feasibility of the operation of a gas core reactor, problems of confining the fissionable material, radiant heating of the propellant, and reactor dynamics and control have been resolved only at the levels of theoretical estimates and experimental investigations of various physical phenomena. Most experiments on hydrodynamic means of confining fissionable materials and stabilizing various types of instability were carried out with liquids and gases in conditions of constant physical parameters. Unfortunately, there are no experimental data on the reactor physics of a system where a significant proportion of the thrust is 
produced by gaseous fissionable material, nor are there experimental data on heating of a gas flow by radiation from a central zone at prototypic engine conditions. The absence of a prototypical experiment which would model the main thermal and hydrodynamic processes and demonstrate the possibility of confining the uranium and heating the propellant under conditions of a controllable fission reaction make it impossible to reach a final conclusion on the feasibility of a gas core engine.

Major efforts were made beginning in the early 1970s to perform such an experiment. A nonnuclear test was considered, with heating of a gas by a beam of energetic electrons from a special accelerator. This experiment was conceived in great detail, however, it was never implemented due to major technical difficulties in achieving uniform heat release in the uranium with a narrow beam of electrons.

At the same time the possibility of an experiment in an external neutron flux using uranium hexafluoride was considered. However, the small scale of the model and low neutron flux of the available reactor made it impossible to obtain sufficiently high temperatures. This made it impossible to reproduce the thermal and hydrodynamic processes or to demonstrate magnetic confinement.

Gradually it became apparent that realistic model experiments were in fact commensurate in scale and parameters with the engine itself. However, experiments involving the moderator design and auxiliary systems could be much simpler. The principal scientific, technical, and technological data necessary for developing and building such a proof-of-principle demonstration were available. In the end, no such experiment was designed, because a decision to conduct it was not made.

\subsection{Selecting the Design}

The basic engine design adopted in the USSR for continued work was the single-cavity concept with magnetic flow stabilization and a thrust of 220-330 klbf. The main reasons for this choice were:

- For those space missions being proposed, the high thrust multi-cavity design had no distinct advantage over suitably modified, traditional chemical systems.

- The single-cavity design was found to be the only system which could satisfy the requirements for certain missions, most notably, rapid manned flight to Mars.

- The use of magnetic flow stabilization is required to minimize losses of fissionable material during the extended operating time proposed for the engine on a Mars mission. 


\section{COMPARATIVE ASSESSMENT OF SOVIET AND AMERICAN PROGRAMS}

Both programs were begun at about the same time and continued simultaneously according to more or less the same goals: evaluation of individual processes and design work at the conceptual design level. ${ }^{10}$ Also similar was the proposed use of the GCNTP system for space flight to the planets with thrust ranging from 50-200 klbf.

The main difference between the programs was the method of gaseous uranium confinement. Literature known to the author indicates that American scientists had from the outset rejected the idea of using a magnetic field and set their sights instead on using purely hydrodynamic confinement methods. This was perhaps due to fears of specific instabilities, as well as the complexity and large weight of a magnetic system. ${ }^{11}$ Soviet scientists proposed the simultaneous use of both confinement methods.

As the author sees it, both programs had prepared and approached the stage of a realistic modeling experiment in a neutron flux, which requires a very large investment of money. This stage was reached in the middle or late 1970s, and the lack of desire to conduct such an experiment led to a gradual reduction in detailed theoretical and experimental studies of the individual physical phenomena.

Going beyond the framework of this report, it is felt that the delay in developing a gas core reactor delays progress on solving a variety of important problems central to advanced systems for propulsion in space and power production on earth. 


\section{REFERENCES}

1. See PERSONAE for identification of scientists mentioned by name in this report.

2. This paper was not published in the open literature.

3. See the ACRONYMS section.

4. Because of other heat losses from the engine, the practical limit is closer to 2,500 sec.

5. V. M. Ievlev, Turbulent Motion of High-Temperature Continuous Media, Nauka, 1975.

6. V. G. Lushchik, Izv., AN SSSR, MZhG, 1, 1972.

7. M. M. Gurfink, Izv., AN SSSR, MZhG, 2, 1973.

8. A. V. Volkov, M. M. Gurfink, and A. P. Poluektov, Magnetic Hydrodynamics, 1, 1976.

9. M. M. Gurfink, DAN SSSR, vol 216, 6, 1974.

10. E. E. Duke and W. J. Houghton, AIAA Preprint N 66-621, AIAA 2nd Propulsion Joint Specialist Conference, June 13-17, 1966.

11. See, however, Eric J. Lerner, Aerospace America, 5, 1990. 

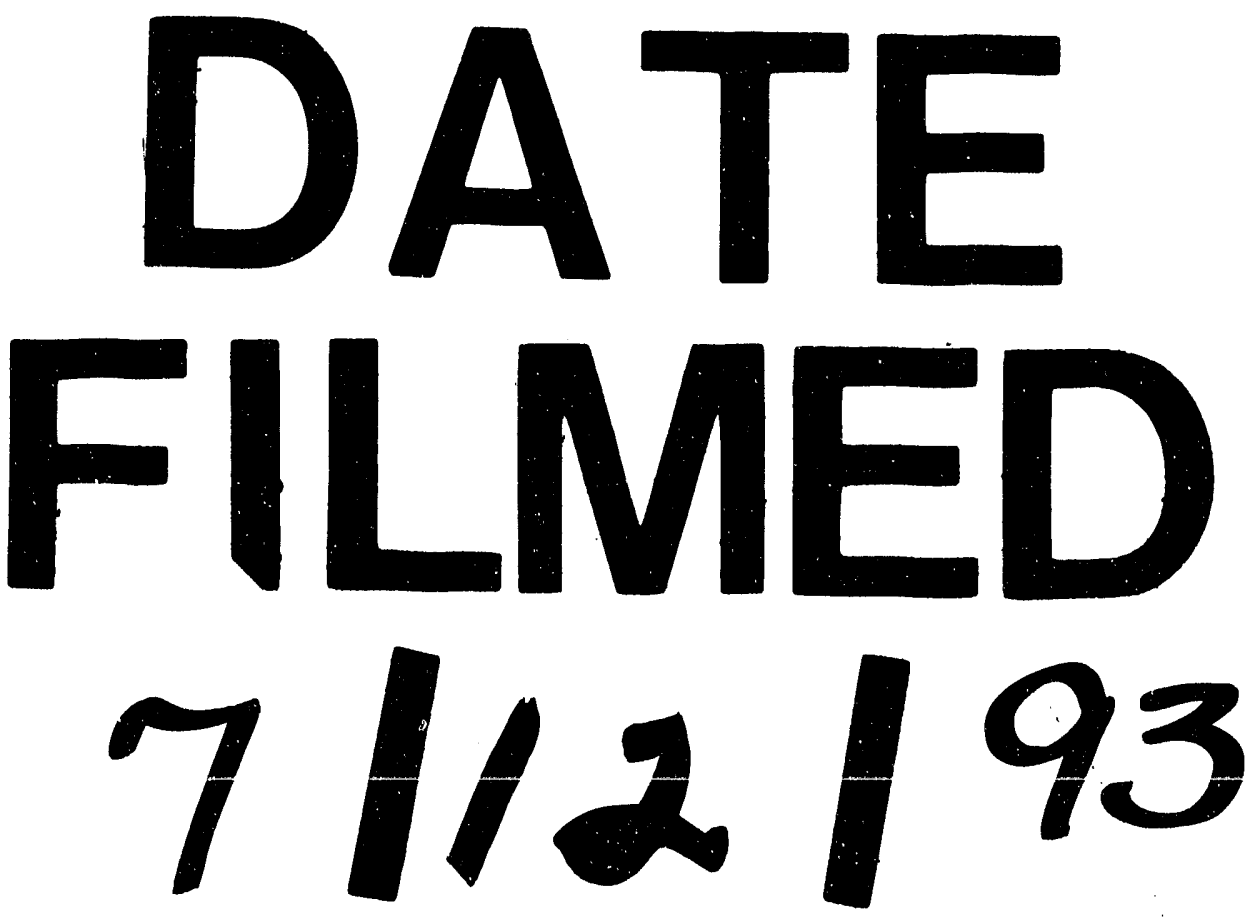
